\title{
Harmonic analysis on semisimple symmetric spaces A survey of some general results
}

\author{
E. P. van den Ban, M. Flensted-Jensen and H. Schlichtkrull
}

\begin{abstract}
We give a survey of the status of some of the fundamental problems in harmonic analysis on semisimple symmetric spaces, including the description of the discrete series, the definition of the Fourier transform, the inversion formula, the Plancherel formula and the PaleyWiener theorem.
\end{abstract}

Key words: Harmonic analysis, Symmetric space, Fourier transform, Discrete series, Plancherel formula, Paley-Wiener theorem

\section{Introduction}

The rich and beautiful theory of harmonic analysis on $\mathbb{R}$ and $\mathbb{T}=(\mathbb{R} / \mathbb{Z})$ has become a powerful tool, widely used in other branches of mathematics, in physics, engineering etc. From our point of view all the basic questions are completely and explicitly solved: The Fourier transform is defined, there exists a Plancherel formula and an inversion formula for it, and (for $\mathbb{R}$ ) there is a Paley-Wiener theorem, describing the image of the space of smooth compactly supported functions.

There exist many generalizations of this theory. Let us mention a few of these, based on various ways of viewing the exponential function $x \mapsto e^{\lambda x}$ on $\mathbb{R}(\lambda \in i \mathbb{R})$ and on $\mathbb{T}(\lambda \in 2 \pi i \mathbb{Z})$ :

- On $\mathbb{R}$, the exponential functions are eigenfunctions for $d / d x$ : Spectral theory for differential operators. Sturm-Liouville theory. Expansion in orthogonal polynomials.

- The exponential functions are characters for the topological groups $\mathbb{R}, \mathbb{T}$ : Fourier analysis on locally compact Abelian groups. The Peter-Weyl theory for Fourier analysis on compact groups.

- The exponential functions generate one dimensional representations of the Lie groups $\mathbb{R}, \mathbb{T}$ : The representation theory for compact Lie groups (the Cartan-Weyl classification, Weyl's character formula etc.). Representation theory for general Lie groups (semisimple, reductive, nilpotent, solvable etc.).

- The manifolds $\mathbb{R}, \mathbb{T}$ are homogeneous spaces for the Lie groups $\mathbb{R}$ and $\mathbb{T}$, respectively (the action being translation), and the exponential functions 
are simultaneous eigenfunctions for the algebras of invariant differential operators on these manifolds: Harmonic analysis on homogeneous spaces of Lie groups.

As an example of the last point we could mention the theory of spherical harmonic expansion on the $n$-sphere $S^{n}$, which is a homogeneous space for the rotation group $O(n+1)$. The spherical harmonics are eigenfunctions for the Laplace operator, which is rotation invariant.

Here we take this last mentioned viewpoint. We claim that inside the class of smooth manifolds the class of (not necessarily Riemannian) reductive symmetric spaces constitutes an appropriate framework for generalization of harmonic analysis: On the one hand this class of manifolds is wide enough to contain very many important spaces of relevance in other branches of mathematics and in physics. On the other hand it is restrictive enough to make feasible a theory of harmonic analysis, with explicit parametrizations and descriptions of representations, explicit Plancherel formulae, etc. The irreducible members of the class of reductive symmetric spaces are either one-dimensional flat, i.e. $\mathbb{R}$ or $\mathbb{T}$, or semisimple. In this paper we discuss the semisimple symmetric spaces. The exposition in the present paper consists of a rewriting and updating of parts of [8], extended with a description of recent developments.

\section{Semisimple symmetric spaces}

\subsection{DEFinition AND STRUCTURE}

We define a semisimple symmetric space as follows:

Definition. A homogeneous space $M=G / H$ is called a semisimple symmetric space if $G$ is a connected semisimple Lie group and $H$ an open subgroup of the group of fixed points for an involution $\sigma$ of $G$.

We are only going to introduce the most necessary aspects and technicalities of the general theory of semisimple symmetric spaces. For a more complete treatment and some of the details we refer to [33], [60], [41, Part II] and the references cited there.

An important case is when $M$ is a semisimple Lie group $G_{1}$, i.e. when $G$ is the product $G_{1} \times G_{1}$ and its action on $G_{1}$ is the left times right action. The involution of $G$ is given by $\sigma(x, y)=(y, x)$, and $H$ is the diagonal $d\left(G_{1}\right)$. We shall call this the group case.

Our goal in this paper is to indicate the state of the art for harmonic analysis on semisimple symmetric spaces. From now on we assume that $M=G / H$ is such a space.

For simplicity of exposition we assume (which we may up to coverings of $M$ ) that $G$ is a closed subgroup of $\operatorname{GL}(n, \mathbb{R})$ for some $n$, and that $G$ is stable under transposition. Let $K=G \cap \mathrm{SO}(n)$, or equivalently $K=G^{\theta}$, where $\theta(x)={ }^{t} x^{-1}$, 
then $K$ is a maximal compact subgroup of $G$. We may choose the base point such that $\theta(H)=H$, or equivalently, such that $\sigma \circ \theta=\theta \circ \sigma$.

We shall distinguish between the following 3 types of irreducible semisimple symmetric spaces:

- $M$ is of the compact type if $G=K$, or equivalently if all geodesic curves have compact closures.

- $M$ is of the non-compact type if $H=K$, or equivalently if all geodesic curves have non-compact closures.

- $M$ is of the non-Riemannian type if $G \neq K$ and $K \neq H$, or equivalently if there exist geodesic curves of both types.

If $M$ is of one of the first two types we say that it is of the Riemannian type, because it then has a natural structure as a Riemannian manifold. In the third case the natural structure is only pseudo-Riemannian. Notice that a simple group $G_{1}$, considered as a symmetric space, is either of the compact type or of the nonRiemannian type.

\subsection{EXAMPLES}

The irreducible symmetric spaces have been classified by M. Berger [17]. Compared with the list of Riemannian symmetric spaces (see [45, Ch.X]), Berger's list is considerably longer.

There is (up to coverings) one two-dimensional space of each of the three types:

- The compact type: The $2-$ sphere $\mathrm{S}^{2}=\mathrm{SO}(3) / \mathrm{SO}(2)$.

- The non-compact type: The hyperbolic 2-space $M=\mathrm{H}^{2}$. This has several isomorphic realizations: $\operatorname{As~} \mathrm{SL}(2, \mathbb{R}) / \mathrm{SO}(2)$, as $\mathrm{SU}(1,1) / \mathrm{S}(\mathrm{U}(1) \times \mathrm{U}(1))$, or as $\mathrm{SO}_{e}(2,1) / \mathrm{SO}(2)$, corresponding to, respectively, the upper half plane in $\mathbb{C}$, the unit disk in $\mathbb{C}$, or a sheet of the two-sheeted hyperboloid in $\mathbb{R}^{3}$.

- The non-Riemannian type: The one-sheeted hyperboloid in $\mathbb{R}^{3}, H^{1,1}=$ $\mathrm{SO}_{e}(2,1) / \mathrm{SO}_{e}(1,1)$, which can also be realized as $\mathrm{SL}(2, \mathbb{R}) / \mathrm{SO}(1,1)$. It has the two-fold cover $\mathrm{SL}(2, \mathbb{R}) / \mathrm{SO}_{e}(1,1)$.

In higher dimensions there exist several 'families' of symmetric spaces, many of which have one of the spaces above as their lowest dimensional member. For example we could mention:

The $n$-sphere: $\mathrm{S}^{n}=\mathrm{SO}(n+1) / \mathrm{SO}(n)$.

The space of positive definite quadratic forms in $\mathbb{R}^{n}: M=\operatorname{SL}(n, \mathbb{R}) / \operatorname{SO}(n)$.

The space of quadratic forms of signature $(p, q)$ in $\mathbb{R}^{n}$, (where $n=p+q$ ):

$M=\mathrm{SL}(n, \mathbb{R}) / \mathrm{SO}(p, q)$.

The hyperboloids in $\mathbb{R}^{n+1}$ :

$M=\mathrm{H}^{p, q}=\left\{x \in \mathbb{R}^{n+1} \mid x_{1}^{2}+\cdots+x_{p}^{2}-x_{p+1}^{2}-\cdots-x_{p+q+1}^{2}=-1\right\}$ where $p+q=n$ (if $q=0$ one must take a connected component). Here $M=\operatorname{SO}_{e}(p, q+1) / \operatorname{SO}_{e}(p, q)$.

Similarly, one can take the corresponding spaces over the complex numbers or over the quaternions. 


\subsection{SOME BASIC NOTATION}

Let $G, H, K, \sigma$ and $\theta$ be as above. Let $\mathfrak{g}$ be the (real) Lie algebra of $G$, and let $\mathfrak{h}$ and $\mathfrak{k}$ be the subalgebras corresponding to $H$ and $K$, and $\mathfrak{q}$ and $\mathfrak{p}$ their respective orthocomplements with respect to the Killing form. Then

$$
\mathfrak{g}=\mathfrak{h} \oplus \mathfrak{q}=\mathfrak{k} \oplus \mathfrak{p}
$$

is the decomposition of $\mathfrak{g}$ into the \pm 1 eigenspaces for $\sigma$ and $\theta$ respectively. Since $\theta$ and $\sigma$ commute we also have the joint decomposition

$$
\mathfrak{g}=\mathfrak{h} \cap \mathfrak{k} \oplus \mathfrak{h} \cap \mathfrak{p} \oplus \mathfrak{q} \cap \mathfrak{k} \oplus \mathfrak{q} \cap \mathfrak{p}
$$

Notice that there is a natural identification of $\mathfrak{q}$ with the tangent space $T_{x_{o}}(M)$ at the base point $x_{o}=e H$. We denote by $\mathfrak{g} \mathbb{C}, \mathfrak{h} \mathbb{C}$ etc. the complexifications of $\mathfrak{g}, \mathfrak{h}$ etc.

A Cartan subspace $\mathfrak{b}$ for $G / H$ is a maximal Abelian subspace of $\mathfrak{q}$, consisting of semisimple elements. (If we assume, as we may in the following, that $\mathfrak{b}$ is $\theta$-invariant, then all its elements are automatically semisimple, once $\mathfrak{b}$ is maximal Abelian). All Cartan subspaces have the same dimension, which we call the rank of $M$. The number of $H$-conjugacy classes of Cartan subspaces is finite. Geometrically, a Cartan subspace is the tangent space of a maximally flat regular subsymmetric space.

We say that a Cartan subspace $\mathfrak{b}$ is fundamental if the intersection $\mathfrak{b} \cap \mathfrak{k}$ is maximal Abelian in $\mathfrak{q} \cap \mathfrak{k}$, and that it is maximal split if the intersection $\mathfrak{b} \cap \mathfrak{p}$ is maximal Abelian in $\mathfrak{q} \cap \mathfrak{p}$. There is, up to conjugation by $K \cap H$, a unique fundamental and a unique maximal split Cartan subspace. If the fundamental Cartan subspace is contained in $\mathfrak{k}$ it is called a compact Cartan subspace. The dimension of the $\mathfrak{p}$-part of a maximal split Cartan subspace is called the split rank of $M$.

Let $\mathbb{D}(G / H)$ denote the algebra of $G$-invariant differential operators on $G / H$. There is a natural isomorphism (the Harish-Chandra isomorphism) $\chi$ of this algebra with the algebra $S(\mathfrak{b})^{W}$ of $W$-invariant elements in the symmetric algebra of any Cartan subspace $\mathfrak{b}_{\mathbb{C}}$. Here $W$ is the reflection group of the root system of $\mathfrak{b}_{\mathbb{C}}$ in $\mathfrak{g} \mathbb{C}$. In particular, $\mathbb{D}(G / H)$ is commutative, and its characters are parametrized up to $W$-conjugation by $D \mapsto \chi_{\lambda}(D)=\chi(D)(\lambda) \in \mathbb{C}$. It is known (see [2]) that the symmetric elements of $\mathbb{D}(G / H)$ have self-adjoint closures as operators on $L^{2}(G / H)$.

\section{Basic harmonic analysis}

\subsection{HARMONIC ANALYSIS ON $\mathbb{R}^{n}$}

We want to generalize the basic notions and results from harmonic analysis on $\mathbb{R}^{n}$. These are:

The Fourier transform: $f \mapsto f^{\wedge}(\lambda)=(2 \pi)^{-n / 2} \int_{\mathbb{R}^{n}} f(t) e^{-i \lambda \cdot t} d t, f \in C_{c}^{\infty}\left(\mathbb{R}^{n}\right)$. 
The inversion formula: If $f \in C_{c}^{\infty}\left(\mathbb{R}^{n}\right)$ then

$$
f(x)=(2 \pi)^{-n / 2} \int_{\mathbb{R}^{n}} f^{\wedge}(\lambda) e^{i \lambda \cdot x} d \lambda .
$$

The Plancherel theorem: $f \mapsto f^{\wedge}$ extends to an isometry of $L^{2}\left(\mathbb{R}^{n}\right)$ onto $L^{2}\left(\mathbb{R}^{n}\right)$.

The Paley-Wiener theorem: $f \mapsto f^{\wedge}$ is a bijection of $C_{c}^{\infty}\left(\mathbb{R}^{n}\right)$ onto $\mathrm{PW}\left(\mathbb{R}^{n}\right)$, where $\mathrm{PW}\left(\mathbb{R}^{n}\right)$ is the space of rapidly decreasing entire functions of exponential type. More precisely, a complex function $\psi$ on $\mathbb{R}^{n}$ belongs to $\mathrm{PW}\left(\mathbb{R}^{n}\right)$ if and only if it extends to an entire function on $\mathbb{C}^{n}$ for which there exists $R>0$ such that the following holds for all $N \in \mathbb{N}$ :

$$
\sup _{\lambda \in \mathbb{C}^{n}}(1+|\lambda|)^{N} e^{-R|\operatorname{Im} \lambda|}|\psi(\lambda)|<+\infty
$$

The aim of the basic harmonic analysis on $G / H$ is to obtain analogues of these notions and results.

\subsection{THE 'ABSTRACT' HARMONIC ANALYSIS ON A SEMISIMPLE SYMMETRIC SPACE}

Let $G$ and $H$ be as above, then $M=G / H$ has an invariant measure, and the action of $G$ by translations gives a unitary representation $\mathcal{L}$ in the associated Hilbert space $L^{2}(G / H)$. From general representation theory it is known (since $G$ is 'type 1 ') that this representation can be decomposed as a direct integral of irreducible unitary representations:

$$
\mathcal{L} \simeq \int_{G^{\wedge}}^{\oplus} m_{\pi} \pi d \mu(\pi),
$$

where the measure $d \mu$ (whose class is uniquely determined) is called the Plancherel measure, and $m_{\pi}$ (which is unique almost everywhere) the multiplicity of $\pi$. Moreover, only the so-called $H$-spherical representations can occur in this decomposition. By definition, an irreducible unitary representation $\left(\pi, \mathcal{H}_{\pi}\right)$ of $G$ is $H$-spherical if the space $\left(\mathcal{H}_{\pi}^{-\infty}\right)^{H}$ of its $H$-fixed distribution vectors is non-trivial. Here we denote by $\mathcal{H}_{\pi}^{\infty}$ and $\mathcal{H}_{\pi}^{-\infty}$, respectively the $C^{\infty}$ and the distribution vectors for $\mathcal{H}_{\pi}$, such that $\mathcal{H}_{\pi}^{\infty} \subset \mathcal{H}_{\pi} \subset \mathcal{H}_{\pi}^{-\infty}$. We write

$$
\mathcal{V}_{\pi}=\left(\mathcal{H}_{\pi}^{-\infty}\right)^{H}
$$

It is known (see [2]) that $m_{\pi} \leq \operatorname{dim} \mathcal{V}_{\pi}<+\infty$, in particular, all multiplicities are finite. Denote by $G_{H}^{\wedge}$ the set of (equivalence classes) of $H$-spherical representations, then it follows that the Plancherel measure $d \mu$ is carried by $G_{H}^{\wedge}$.

The 'abstract' Fourier transform $f \mapsto f^{\wedge}(\pi)$ for $G / H$ is now defined by

$$
f^{\wedge}(\pi)(\eta)=\pi(f) \eta=\int_{G / H} f(x) \pi(x) \eta d x \in \mathcal{H}_{\pi}^{\infty}
$$


for $\pi \in G_{H}^{\wedge}, \eta \in \mathcal{V}_{\pi}$ and $f \in C_{c}^{\infty}(G / H)$. Thus

$$
f^{\wedge}(\pi) \in \operatorname{Hom} \mathbb{C}\left(\mathcal{V}_{\pi}, \mathcal{H}_{\pi}^{\infty}\right) \simeq \mathcal{H}_{\pi}^{\infty} \otimes \mathcal{V}_{\pi}^{*}
$$

(notice that the integral over $G / H$ only makes sense because $\eta$ is $H$-invariant). One can prove (using [57] and [62]) that there exists for almost all $\pi \in G_{H}^{\wedge}$ a subspace $\mathcal{V}_{\pi}^{o}$ (of dimension $m_{\pi}$ ) of $\mathcal{V}_{\pi}$, equipped with the structure of a Hilbert space, such that if $f^{\wedge}(\pi)$ is restricted to $\mathcal{V}_{\pi}^{o}$ for almost all $\pi$, then $f \mapsto f^{\wedge}$ extends to an isometry of $L^{2}(G / H)$ onto $\int_{G_{H}^{\wedge}}^{\oplus} \operatorname{Hom} \mathbb{C}\left(\mathcal{V}_{\pi}^{o}, \mathcal{H}_{\pi}\right) d \mu(\pi)$. Here the norm on $\operatorname{Hom} \mathbb{C}\left(\mathcal{V}_{\pi}^{o}, \mathcal{H}_{\pi}\right)$ is given by

$$
\|\varphi\|_{\pi}^{2}=\operatorname{Tr}\left(\varphi^{*} \circ \varphi\right)=\sum_{i}\left\|\varphi\left(v_{i}\right)\right\|^{2}, \quad \varphi \in \operatorname{Hom} \mathbb{C}\left(\mathcal{V}_{\pi}^{o}, \mathcal{H}_{\pi}\right)
$$

where $\varphi^{*}$ is the adjoint of $\varphi$ and $\left\{v_{i}\right\}_{i=1, \ldots, m_{\pi}}$ is an orthonormal basis in $\mathcal{V}_{\pi}^{o}$.

We thus have the Plancherel formula

$$
\|f\|_{2}^{2}=\int_{G_{H}^{\wedge}}\left\|f^{\wedge}(\pi)\right\|_{\pi}^{2} d \mu(\pi), \quad f \in L^{2}(G / H)
$$

Similarly, there is the inversion formula (for suitably nice functions $f$ )

$$
f(e)=\int_{G_{H}^{\wedge}} \sum_{i=1}^{m_{\pi}}\left\langle f^{\wedge}(\pi) v_{i} \mid v_{i}\right\rangle d \mu(\pi)
$$

(Here $\langle\cdot \mid \cdot\rangle$ denotes the inner product on $\mathcal{H}_{\pi}$, as well as the naturally associated pairing $\mathcal{H}_{\pi}^{\infty} \times \mathcal{H}_{\pi}^{-\infty} \rightarrow \mathbb{C}$.) Consequently we also have, for suitable $f$

$$
f(x)=\int_{G_{H}} \sum_{i=1}^{m_{\pi}}\left\langle f^{\wedge}(\pi) v_{i} \mid \pi(x) v_{i}\right\rangle d \mu(\pi) .
$$

The basic problems are now

(a) Describe (parametrize) $G_{H}^{\wedge}$, or at least $\mu$-almost all of it.

(b) For $\mu$-almost all $\pi \in G_{H}^{\wedge}$ describe (parametrize) $\mathcal{V}_{\pi}^{o}$ and its Hilbert space structure.

(c) Determine $d \mu$ explicitly.

A Paley-Wiener theorem would amount to an intrinsic description of the Fourier image of $C_{c}^{\infty}(G / H)$ in terms of $G_{H}^{\wedge}$. We add this as a fourth basic problem:

(d) Describe $C_{c}^{\infty}(G / H)^{\wedge}$ in terms of the parametrizations and possible holomorphic extensions. 
For each $\pi \in G_{H}^{\wedge}$ we have that $\mathcal{V}_{\pi}$ is a $\mathbb{D}(G / H)$-module in a natural way. Using that the symmetric elements of $\mathbb{D}(G / H)$ are essentially selfadjoint operators on $L^{2}(G / H)$ one can show (with the arguments in [62]) that $\mathcal{V}_{\pi}^{o}$ can be chosen to be invariant and diagonalizable for this action. Thus $\mathcal{V}_{\pi}^{o}$ is spanned by its joint eigenvectors for $\mathbb{D}(G / H)$. Let $\mathfrak{b} \subset \mathfrak{q}$ be a Cartan subspace. Then such an eigenvector satisfies

$$
\pi(D) v=\chi_{\lambda}(D) v, \quad D \in \mathbb{D}(G / H),
$$

for some $\lambda \in \mathfrak{b}_{\mathbb{C}}^{*}$. We say that $v$ is a spherical vector of type $\lambda$, and that the orthonormal basis $\left\{v_{i}\right\}_{i=1, \ldots, m_{\pi}}$ in $\mathcal{V}_{\pi}^{o}$ is spherical if its members are spherical.

The maps $\xi_{\pi, i}: f \mapsto\left\langle f^{\wedge}(\pi) v_{i} \mid v_{i}\right\rangle$ in (4) are $H$-invariant distributions on $G / H$. As distributions on $G$ they are positive definite and extreme (see [62]). With a spherical basis $\left\{v_{i}\right\}$ each $\xi_{\pi, i}$ is also a spherical distribution, that is, an $H$-invariant eigendistribution for $\mathbb{D}(G / H)$. The solution of Problem (b) is then closely related to the study of the spherical distributions.

\subsection{RESUlts FOR SPECIFIC ClASSES OF SYMMETRIC SPACES}

Here we give some brief remarks concerning the above problems for some specific classes of semisimple symmetric spaces.

3.3.1. The compact type. For a homogeneous space $G / H$ with a compact group $G$ the abstract formulation above follows easily from the Peter-Weyl theorem and the Schur orthogonality relations. In particular, $\mathcal{V}_{\pi}^{o}=\mathcal{V}_{\pi}=\mathcal{H}_{\pi}^{H}$, and if we give $\mathcal{V}_{\pi}^{o}$ the subspace norm from $\mathcal{H}_{\pi}$, we have $d \mu(\pi)=\operatorname{dim}(\pi)$. For the symmetric spaces of compact type we then have the following explicit solutions to the above problems (see $[26],[46, \S \mathrm{V} .4])$ :

(a) $G_{H}^{\wedge}$ is parametrized by a subset of the set of dominant weights.

(b) $\operatorname{dim} \mathcal{V}_{\pi}^{o}=1$ for $\pi \in G_{H}^{\wedge}$.

(c) $d \mu$ is given by Weyl's dimension formula.

(d) The smooth functions are determined by a certain growth condition on the Fourier transforms (see [61]).

3.3.2. The non-compact type. We write $M$ as $G / K$. The four questions were settled beautifully by the work of Harish-Chandra, Helgason and others. See [46, $\S$ IV.7] and $[47$, Ch. III]. Let $\mathfrak{a}$ be a maximal Abelian subspace of $\mathfrak{p}$.

(a) A sufficient subset of $G_{K}^{\wedge}$ is parametrized (up to conjugacy by the Weyl group) by means of the spherical functions $\varphi_{\lambda}, \lambda \in i \mathfrak{a}^{*}$ and the corresponding spherical principal series representations $\left(\pi_{\lambda}, \mathcal{H}_{\lambda}\right)$.

(b) For $\pi=\pi_{\lambda} \in G_{K}^{\wedge}$ we have $\mathcal{V}_{\pi}^{o}=\mathcal{H}_{\lambda}^{K}$ and $\operatorname{dim}\left(\mathcal{V}_{\pi}^{o}\right)=1$. We can then use the subspace norm from $\mathcal{H}_{\lambda}$.

(c) The Plancherel measure is given by $d \mu\left(\pi_{\lambda}\right)=|\mathbf{c}(\lambda)|^{-2} d \lambda$ on $i \mathfrak{a}^{*} / W$. Here $\mathbf{c}(\lambda)$ is Harish-Chandra's $c$-function, which is explicitly given in terms of the structure of $G / K$ by the formula of Gindikin-Karpelevic. 
(d) We have $C_{c}^{\infty}(K \backslash G / K)^{\wedge}=\operatorname{PW}(\mathfrak{a})^{W}$. Here $\mathrm{PW}(\mathfrak{a})^{W}$ is the space of $W-$ invariant functions in the image space $\mathrm{PW}(\mathfrak{a})$ for the Fourier transform

$$
f \mapsto f^{\wedge}(\lambda)=\int_{\mathfrak{a}} f(X) e^{-\lambda(X)} d X, \quad \lambda \in \mathfrak{a}_{\mathbb{C}}^{*}, f \in C_{c}^{\infty}(\mathfrak{a}),
$$

that is, the space of rapidly decreasing entire functions of exponential type on $\mathfrak{a}_{\mathbb{C}}^{*}$ (see Section 3.1, but note that since the imaginary unit $i$ is not present in the exponent in (5), one has to replace $\operatorname{Im} \lambda$ by $\operatorname{Re} \lambda$ in (2)). Helgason has extended the Paley-Wiener theorem to the space $C_{c}^{\infty}(K ; G / K)$ of $K-$ finite functions in $C_{c}^{\infty}(G / K)$, and also to the full space $C_{c}^{\infty}(G / K)$. See [47, Ch. III, Thms. 5.1, 5.11].

3.3.3. The group case, $M=G_{1}$. This case is settled by the work of Harish-Chandra ([39]) and others (for expositions, see e. g. [48], [64]).

(a) The map $\pi_{1} \mapsto \pi_{1} \otimes \pi_{1}^{*}$ is a bijective correspondence from the unitary dual $G_{1}^{\wedge}$ onto $G_{H}^{\wedge}$. A sufficient subset of $G_{1}^{\wedge}$ is described by the discrete series and different families of (cuspidal) principal series.

(b) For $\pi_{1} \in G_{1}^{\wedge}$ and $\pi=\pi_{1} \otimes \pi_{1}^{*}$ we have $\mathcal{V}_{\pi}=\left(\mathcal{H}_{\pi}^{-\infty}\right)^{H}=\mathbb{C} 1_{\pi_{1}}$, where $1_{\pi_{1}}$ is the identity operator on $\mathcal{H}_{\pi_{1}}$. Notice however that in this case $\mathcal{V}_{\pi} \not \subset \mathcal{H}_{\pi}$, since the latter space can be identified with the space of Hilbert-Schmidt operators on $\mathcal{H}_{\pi_{1}}$. We take $\mathcal{V}_{\pi}^{o}=\mathcal{V}_{\pi}$, and use on it the Hilbert space structure obtained from the identification with $\mathbb{C}$ in which $1_{\pi_{1}}=1$.

(c) With the above choice one can give $d \mu$ explicitly in terms of the formal degrees of discrete series and certain $c$-functions.

(d) A Paley-Wiener theorem for the $K$-finite functions on $G_{1}$ has been established in [22] (in split rank one) and [1] (in general). In particular, the Paley-Wiener space is determined by the minimal principal series only. The extension of the Paley-Wiener theorem to the full space $C_{c}^{\infty}\left(G_{1}\right)$ is still an open problem.

3.3.4. The non-Riemannian type, rank one. There is an extensive literature dealing with the questions (a)-(c) on specific classes of rank one symmetric spaces of the non-Riemannian type. See for example [31], [62], [52]. Common for all these spaces is that the decomposition of $L^{2}(G / H)$ contains a discrete series as well as a continuous part.

3.3.5. Type $G_{\mathbb{C}} / G_{\mathbb{R}}$. When $G$ is complex and $H$ is a real form of it, precise solutions to questions (a)-(c) have been given by P. Harinck. See [20], [34], [35], [36].

\subsection{RESUlts FOR GENERAL SEMISIMPLE SYMMETRIC SPACES}

The listed basic problems have been solved in a general setting for semisimple symmetric spaces. In the following sections we outline the solution, with precise references to the literature. 
By analogy with the group case one expects in general that the left regular representation $\mathcal{L}$ on $L^{2}(G / H)$ can be decomposed in several 'series' of representations, one series for each $H$-conjugacy class of Cartan subspaces for $\mathfrak{q}$. The most extreme of these would then be the 'most continuous' part, corresponding to the conjugacy class of Cartan subspaces with maximal $\mathfrak{p}$-part (the maximal split Cartan subspaces) and the 'most discrete' part (sometimes called the fundamental series), corresponding to the conjugacy class of Cartan subspaces with maximal $\mathfrak{k}$-part (the fundamental Cartan subspaces). The series corresponding to the remaining conjugacy classes of Cartan subspaces would then be called 'the intermediate series'. If the fundamental Cartan subspaces are compact, then the 'most discrete' part is in fact the discrete series, that is, the irreducible subrepresentations of $\mathcal{L}$.

In fact, this analogy with the group case holds rather precisely, as we shall explain below. In Section 4 we discuss discrete series and in Section 5 the most continuous series. In Sections 6-7 we then discuss the Plancherel and Paley-Wiener theorems for $G / H$.

\section{The discrete series}

The basic existence theorem is the following, where we preserve the notions from above. Let $L_{d}^{2}(G / H) \subset L^{2}(G / H)$ be the closed linear span of the irreducible subrepresentations of $\mathcal{L}$.

Theorem 1, [32], [55]. Let $G / H$ be a semisimple symmetric space. Then the discrete series space $L_{d}^{2}(G / H)$ is non-zero if and only if

$$
\operatorname{rank}(G / H)=\operatorname{rank}(K / K \cap H)
$$

The condition (6) means that $G / H$ has a compact Cartan subspace. An equivalent more geometric formulation is that it has a compact maximally flat subsymmetric space.

In the group case this result reduces to Harish-Chandra's theorem, that the existence of discrete series is equivalent to the existence of a compact Cartan subgroup, cf. [38]. In fact the proof in [32] of the existence part of the theorem is different from Harish-Chandra's proof for the group case, see also [48], where the symmetric space viewpoint has been adapted in the proof for the group case.

We shall now discuss Problems (a), (b) and (c) for the discrete series. Assume (as we may by the above theorem) that (6) holds, and let $\mathfrak{t}$ be a compact Cartan subspace of $\mathfrak{q}$. Let $\Sigma$ be the root system of $\mathfrak{t} \mathbb{C}$ in $\mathfrak{g} \mathbb{C}$ and $\Sigma_{c}$ the subsystem of $\mathfrak{t} \mathbb{C}$ in $\mathfrak{k}_{\mathbb{C}}$. Let $W$ and $W_{c}$ be the corresponding reflection groups.

A rough classification of the discrete series is obtained by means of the commutative algebra $\mathbb{D}(G / H)$. Recall that the characters of $\mathbb{D}(G / H)$ are parametrized by $\mathfrak{t}_{\mathbb{C}}^{*} / W$ via the Harish-Chandra isomorphism $\chi: \mathbb{D}(G / H) \rightarrow S(\mathfrak{t})^{W}$. Let $\mathcal{E}_{\lambda}(G / H)$ 
denote the joint eigenspace for $\mathbb{D}(G / H)$ in $C^{\infty}(G / H)$ corresponding to the character $\chi_{\lambda}$, where $\lambda \in \mathfrak{t}_{\mathbb{C}}^{*}$. Then $\mathcal{E}_{w \lambda}(G / H)=\mathcal{E}_{\lambda}(G / H)$ for all $w \in W$. Since $\mathbb{D}(G / H)$ is commutative and its symmetric elements act as essentially selfadjoint operators on $L^{2}(G / H)$, there is a joint spectral resolution of $L^{2}(G / H)$ for this algebra. The resulting decomposition is $G$-invariant because of the invariance of the elements in $\mathbb{D}(G / H)$. It follows (see [2]) that $L_{d}^{2}(G / H)$ admits an orthogonal $G$-invariant decomposition

$$
L_{d}^{2}(G / H)=\bigoplus_{\lambda} L_{\lambda}^{2}(G / H)
$$

where $L_{\lambda}^{2}(G / H)$ is the closure in $L^{2}(G / H)$ of $L^{2}(G / H) \cap \mathcal{E}_{\lambda}(G / H)$, and where the sum extends over the $W$-orbits in the set of those $\lambda \in \mathfrak{t}_{\mathbb{C}}^{*}$ for which $L_{\lambda}^{2}(G / H)$ is non-trivial. In order to parametrize the discrete series we must then determine this set of $\lambda$ 's, and for each $\lambda$ therein the irreducible subrepresentations of $L_{\lambda}^{2}(G / H)$.

Let $\Lambda \subset i \mathfrak{t}^{*}$ denote the set of elements $\lambda \in i \mathfrak{t}^{*}$ satisfying the following conditions (i)-(iii).

(i) $\langle\lambda, \alpha\rangle \neq 0$ for all $\alpha \in \Sigma$.

Given that (i) holds, let

$$
\Sigma^{+}=\{\alpha \in \Sigma \mid\langle\lambda, \alpha\rangle>0\}
$$

then this is a positive system for $\Sigma$. Put $\Sigma_{c}^{+}=\Sigma^{+} \cap \Sigma_{c}$, and let $\rho$, resp. $\rho_{c}$, be defined as half the sum of the $\Sigma^{+}$-roots, resp. $\Sigma_{c}^{+}$-roots, counted with multiplicities.

(ii) $\lambda+\rho$ is a weight for $T_{H}$, i.e. $e^{\lambda+\rho}$ is well defined on $T_{H}$. Here $T_{H}$ denotes the torus in $G / H$ corresponding to $\mathfrak{t}$ (that is, $T_{H}=T /(T \cap H)$ where $T=\exp \mathfrak{t}$ ).

(iii) $\langle\lambda-\rho, \beta\rangle \geq 0$ for each compact simple $\operatorname{root} \beta$ in $\Sigma^{+}$.

(that $\beta$ is compact means that the root space $\mathfrak{g}_{\mathbb{C}}^{\beta}$ is contained in $\mathfrak{k}_{\mathbb{C}}$ ). Notice that (ii) implies that $\Lambda$ is a discrete subset of $i t^{*}$.

Under the assumption that $\lambda \in \Lambda$ there is a rather simple construction (which we shall outline below) of a $\mathfrak{g}$-invariant subspace $\mathcal{U}_{\lambda, K}$ of $C^{\infty}(K ; G / H)$ (the space of $K$-finite functions in $\left.C^{\infty}(G / H)\right)$, which can be shown to be contained in $L_{\lambda}^{2}(G / H)$. Let $\mathcal{U}_{\lambda}$ denote the closure of $\mathcal{U}_{\lambda, K}$ in $L^{2}(G / H)$, then $\mathcal{U}_{\lambda}$ is a subrepresentation of $L_{\lambda}^{2}(G / H)$. Let $\pi_{\lambda}$ denote this subrepresentation.

For 'large' $\lambda \in \Lambda$, or more precisely if $\left\langle\lambda+\rho-2 \rho_{c}, \alpha\right\rangle \geq 0$ for all $\alpha \in \Sigma_{c}^{+}$, it can be shown by elementary methods that $\mathcal{U}_{\lambda} \neq\{0\}$. For the remaining $\lambda$ 's one has to add a more technical assumption in order to ensure that $\mathcal{U}_{\lambda} \neq\{0\}$. We shall not state this condition here (the condition is stated in [50] together with a proof of its necessity for the non-vanishing of $\mathcal{U}_{\lambda}$. The sufficiency is claimed but not proven in the paper).

Theorem 2, [55], [63]. The discrete series space $L_{d}^{2}(G / H)$ is spanned by the $\mathcal{U}_{\lambda}$ 's with $\lambda \in \Lambda$. Moreover for each $\lambda \in \Lambda$ either the representation $\pi_{\lambda}$ is irreducible 
or $\mathcal{U}_{\lambda}$ is zero, and if $\lambda, \lambda^{\prime} \in \Lambda$ we have $\mathcal{U}_{\lambda^{\prime}}=\mathcal{U}_{\lambda}$ if and only if $\lambda^{\prime}=w \lambda$ for some $w \in W_{c}$.

It follows that if $\lambda \in \mathfrak{t}_{\mathbb{C}}^{*}$ then $L_{\lambda}^{2}(G / H)$ is the sum of those $\mathcal{U}_{w \lambda}$ for which $w \in W$ and $w \lambda \in \Lambda$. In particular it has at most as many components as the order of the quotient $W / W_{c}$.

With this result, Problem (a) is almost solved as regards to the discrete series. It is conjectured that $\pi_{\lambda^{\prime}}$ is unitarily equivalent to $\pi_{\lambda}$ if and only if $\mathcal{U}_{\lambda^{\prime}}=\mathcal{U}_{\lambda}$, or equivalently in view of the above, that the discrete series have multiplicity one in the Plancherel formula. The conjecture is proved for all classical groups $G$, and is only open for a few exceptional cases for very special values of $\lambda$ (see [19]).

Evaluation at the base point in $G / H$ gives rise to an $H$-fixed distribution vector $\eta_{\lambda}$ for $\mathcal{U}_{\lambda}$, for which it is easily seen that we have

$$
f^{\wedge}\left(\pi_{\lambda}\right)\left(\eta_{\lambda}\right)=\mathrm{P}_{\lambda} f, \quad f \in C_{c}^{\infty}(G / H),
$$

where $\mathrm{P}_{\lambda}$ is the orthogonal projection of $L^{2}(G / H)$ onto $\mathcal{U}_{\lambda}$. It follows that if we take $\mathcal{V}_{\pi_{\lambda}}^{o}=\mathbb{C} \eta_{\lambda}$ and use on it the Hilbert space structure obtained from the identification with $\mathbb{C}$ in which $\eta_{\lambda}=1$, then $d \mu\left(\pi_{\lambda}\right)=1$. In other words, the Plancherel measure restricts to the counting measure on the discrete series. This provides the solution to Problems (b) and (c) for the discrete series.

At this point it is however interesting to note the following. Though the discrete series has been parametrized as above, it seems to be an open problem to determine an explicit expression for the spherical distribution $\xi_{\lambda}: f \mapsto\left\langle f^{\wedge}\left(\pi_{\lambda}\right) \eta_{\lambda} \mid \eta_{\lambda}\right\rangle$ on $G / H$ associated to $\eta_{\lambda}$ (or equivalently, for the projection operator $\mathrm{P}_{\lambda}$, which is given by convolution with $\xi_{\lambda}$ ). In the group case one knows that $\xi_{\lambda}$ is given by $d_{\lambda} \Theta_{\lambda}$, where $d_{\lambda}$ is the formal degree and $\Theta_{\lambda}$ the character of $\pi_{\lambda}$ (see $[37, \S 5]$ ), but there is no obvious generalization of this formula.

We shall not try to describe the proofs of the above theorems. However as the construction of $\mathcal{U}_{\lambda, K}$ can be described by quite elementary methods we would like to indicate it.

Let the notation be as above, and recall the decomposition (1) of $\mathfrak{g}$. Let $\mathfrak{g}^{d}$ be the real form of $\mathfrak{g} \mathbb{C}$ given by

$$
\mathfrak{g}^{d}=\mathfrak{h} \cap \mathfrak{k} \oplus i(\mathfrak{h} \cap \mathfrak{p}) \oplus i(\mathfrak{q} \cap \mathfrak{k}) \oplus \mathfrak{q} \cap \mathfrak{p}
$$

where $i$ is the imaginary unit. Assume (again for simplicity of exposition) that $G$ is a real form of a linear complex Lie group $G_{\mathbb{C}}$, and let $G^{d}$ be the real form of $G_{\mathbb{C}}$ whose Lie algebra is $\mathfrak{g}^{d}$. Then the subgroup $K^{d}=G^{d} \cap H_{\mathbb{C}}$ is a maximal compact subgroup. The Riemannian symmetric space $G^{d} / K^{d}$ is called the non-compact Riemannian form of $G / H$. The subgroup $H^{d}=G^{d} \cap K_{\mathbb{C}}$ of $G^{d}$ is a (in general non-compact) real form of $K \mathbb{C}$. Let $\left(G \cap G^{d}\right)_{e}$ denote the identity component of $G \cap G^{d}$. Then both $G$ and $G^{d}$ are contained in the set $K \mathbb{C}\left(G \cap G^{d}\right)_{e} H_{\mathbb{C}}$. The $K-$ finite functions on $G / H$ extend naturally to left $K \mathbb{C}$-finite and right $H \mathbb{C}_{\mathbb{C}}$-invariant 
functions on this set (and so do the $H^{d}$-finite functions on $G^{d} / K^{d}$, provided the $H^{d}$-action admits a holomorphic extension to $\left.K_{\mathbb{C}}\right)$. We call this partial holomorphic extension. Let $C^{\infty}(K ; G / H)$ and $C^{\infty}\left(H^{d} ; G^{d} / K^{d}\right)$ be the spaces of $K$-finite, resp. $H^{d}$-finite smooth functions on $G / H$, resp. $G^{d} / K^{d}$. There is a natural action of $\mathfrak{g} \mathbb{C}$ on both of these spaces.

Theorem 3, [32]. Partial holomorphic extension defines a $\mathfrak{g} \mathbb{C}$-equivariant linear injection $f \rightarrow f^{r}$ of $C^{\infty}(K ; G / H)$ into $C^{\infty}\left(H^{d} ; G^{d} / K^{d}\right)$, the image of which is the set of functions in $C^{\infty}\left(H^{d} ; G^{d} / K^{d}\right)$ for which the $H^{d}$-action extends holomorphically to $K \mathbb{C}$. Moreover, $f$ is a joint eigenfunction for $\mathbb{D}(G / H)$ if and only if $f^{r}$ is a joint eigenfunction for $\mathbb{D}\left(G^{d} / K^{d}\right)$.

As an example it is quite easily seen in the group case that $G^{d}=\left(G_{1}\right) \mathbb{C}, H^{d}=$ $\left(K_{1}\right) \mathbb{C}$ and $K^{d}=U_{1}$, where $K_{1}$ is a maximal compact subgroup in $G_{1}$ and $U_{1}$ a compact real form of $\left(G_{1}\right) \mathbb{C}$ containing $K_{1}$.

The construction of $G^{d} / K^{d}$ and Theorem 3 hold independent of assumption (6). However, the latter assumption is crucial for the following construction.

Since $G^{d} / K^{d}$ is a Riemannian symmetric space the joint eigenfunctions for the algebra $\mathbb{D}\left(G^{d} / K^{d}\right)$ can be described by means of the so-called generalized Poisson transform. This is defined as follows. It follows from the fact that $\mathfrak{t}$ is a maximal Abelian subspace of $\mathfrak{q}$, that $\mathfrak{t}^{r}=i \mathfrak{t}$ is a maximal Abelian split subspace for $\mathfrak{g}^{d}$. Hence there is an Iwasawa decomposition

$$
G^{d}=K^{d} T^{r} N^{d}
$$

of $G^{d}$ with $T^{r}=\exp \mathfrak{t}^{r}$, which corresponds to a given $\Sigma^{+}$. Let $P^{d}=M^{d} T^{r} N^{d}$ be the corresponding minimal parabolic subgroup in $G^{d}$, and for $\lambda \in \mathfrak{t}_{\mathbb{C}}^{*}$ let $\mathcal{D}_{\lambda}^{\prime}=$ $\mathcal{D}_{\lambda}^{\prime}\left(G^{d} / P^{d}\right)$ be the space of $(\lambda-\rho)$-homogeneous distributions on $G^{d} / P^{d}$, that is the space of generalized functions $f$ on $G^{d}$ satisfying

$$
f(\text { gman })=a^{\lambda-\rho} f(g), \quad g \in G^{d}, m \in M^{d}, a \in T^{r}, n \in N^{d} .
$$

The group $G^{d}$ acts from the left on this space. The Poisson transform $\mathcal{P}_{\lambda}: \mathcal{D}_{\lambda}^{\prime} \rightarrow$ $C^{\infty}\left(G^{d} / H^{d}\right)$ is defined by

$$
\mathcal{P}_{\lambda} f(x)=\int_{K^{d}} f(x k) d k=\int_{K^{d}} p_{\lambda}(x, k) f(k) d k, \quad x \in G^{d} .
$$

Here the 'Poisson kernel' $p_{\lambda} \in C^{\infty}\left(G^{d} \times K^{d}\right)$ is defined by $p_{\lambda}(x, k)=a^{-\lambda-\rho}$, where $a \in T^{r}$ is the $T^{r}$-part of $x^{-1} k$ in the decomposition (8). It is known that $\mathcal{P}_{\lambda}$ is a $G^{d}$-equivariant injective transformation into a joint eigenspace for $\mathbb{D}\left(G^{d} / K^{d}\right)$ in $C^{\infty}\left(G^{d} / K^{d}\right)$ if $\Sigma^{+}$is given by $(7)$, see e.g. [47, $\S$ II.3.4].

The non-vanishing of $\mathcal{U}_{\lambda}$ for 'large' $\lambda \in \Lambda$ follows by a simple construction of an element $\psi_{\lambda}$ in $\mathcal{U}_{\lambda, K}$ involving the following formula and Theorem 3 :

$$
\psi_{\lambda}^{r}(x)=\int_{K \cap H} p_{\lambda}(x, k) d k, \quad x \in G^{d}
$$


see $[32]$ or $[33]$.

Let $\mathcal{D}_{\lambda, H^{d}}^{\prime}$ be the set of $H^{d}$-finite elements in $\mathcal{D}_{\lambda}^{\prime}$, and let $\mathcal{D}_{\lambda, H^{d}}^{\prime}\left(H^{d} P^{d}\right)$ denote the subset of elements supported on the $H^{d}$-orbit $H^{d} P^{d}$ in $G^{d} / P^{d}$ (which is closed, cf. [49] or [60, Prop. 7.1.8]). Let now $\lambda \in \Lambda$. Then condition (ii) implies that the $H^{d}$-finite action on $\mathcal{D}_{\lambda, H^{d}}^{\prime}\left(H^{d} P^{d}\right)$ extends to a holomorphic $K_{\mathbb{C}^{-}}$action. The space $\mathcal{U}_{\lambda, K}$ is now defined by

$$
\mathcal{U}_{\lambda, K}=\left\{f \in C^{\infty}(K ; G / H) \mid f^{r} \in \mathcal{P}_{\lambda}\left(\mathcal{D}_{\lambda, H^{d}}^{\prime}\left(H^{d} P^{d}\right)\right)\right\} .
$$

The proof that $\mathcal{U}_{\lambda, K} \subset L_{\lambda}^{2}(G / H)$ can be found in [55] (see also [9, Thm. 19.1]).

\section{The most continuous part of $L^{2}(G / H)$}

In this section we discuss Problems (a), (b) and (c) for the 'most continuous part' of $L^{2}(G / H)$ (to be defined below). The main references are [11] and [13].

\subsection{THE Fourier TRANSForm}

Let notation be as in Section 2. In [11], [13] the assumptions on $G / H$ are somewhat more general, but for the sake of exposition we shall not discuss this further. The representations $\pi_{\xi, \lambda}$ that occur in the most continuous part of $L^{2}(G / H)$ are constructed as follows. Let $P=M A N$ be a parabolic subgroup of $G$, with the indicated Langlands decomposition, satisfying $\sigma \theta P=P$ and being minimal with respect to this condition. Then $M$ and $A$ are $\sigma$-stable. Let $\mathfrak{a}_{\mathfrak{q}}=\mathfrak{a} \cap \mathfrak{q}$, where $\mathfrak{a}$ is the Lie algebra of $A$, then it follows that $\mathfrak{a}_{\mathfrak{q}}$ is a maximal Abelian subspace of $\mathfrak{p} \cap \mathfrak{q}$, and that the Levi part $M A$ of $P$ is the centralizer of $\mathfrak{a}_{\mathrm{q}}$ in $G$. Let $\left(\xi, \mathcal{H}_{\xi}\right) \in M_{\text {fu }}^{\wedge}$, the set of (equivalence classes of ) finite dimensional irreducible unitary representations of $M$, and let $\lambda \in i \mathfrak{a}^{*}$. We require that $\lambda \in i \mathfrak{a}_{\mathrm{q}}^{*}$, that is that $\lambda$ vanishes on $\mathfrak{a} \cap \mathfrak{h}$. Then by definition $\pi_{\xi, \lambda}$ is the induced representation $\pi_{P, \xi, \lambda}=\operatorname{Ind}_{P=M A N}^{G} \xi \otimes e^{\lambda} \otimes 1$ (the 'principal series' for $G / H$ ), that is, the representation space $\mathcal{H}_{\xi, \lambda}$ consists of (classes of) $\mathcal{H}_{\xi}$-valued measurable functions $f$ on $G$, square integrable on $K$ and satisfying

$$
f(\text { gman })=a^{-\lambda-\rho} \xi(m)^{-1} f(g), \quad(g \in G, m \in M, a \in A, n \in N),
$$

and $G$ acts from the left. Here $\rho=\frac{1}{2} \operatorname{Tr}_{A_{\mathfrak{n}}} \in \mathfrak{a}_{\mathrm{q}}^{*}$. (The convention in (9) differs from the above cited references: The induction takes place on the opposite side.) The representations $\pi_{\xi, \lambda}$ are irreducible for almost all $\lambda \in i \mathfrak{a}_{\mathrm{q}}^{*}$ by Bruhat's theorem (see [6, Thm. 2.6]).

The Plancherel decomposition for the most continuous part of $L^{2}(G / H)$ is obtained by realizing the abstract Fourier transform explicitly for the principal series. This realization is then a partial isometry of $L^{2}(G / H)$ onto the direct integral

$$
\int_{\xi, \lambda}^{\oplus} m_{\xi} \pi_{\xi, \lambda} d \mu(\xi, \lambda) .
$$


The multiplicities $m_{\xi}$ (which happen to be independent of $\lambda$ ) and the measure $d \mu(\xi, \lambda)$ are explicitly described below. The most continuous part of $L^{2}(G / H)$, denoted $L_{\mathrm{mc}}^{2}(G / H)$, is then by definition the orthocomplement of the kernel of this partial isometry. Its Plancherel decomposition is exactly given by (10).

In order to realize the Fourier transform we must first discuss the space $\mathcal{V}_{\xi, \lambda}=$ $\left(\mathcal{H}_{\xi, \lambda}^{-\infty}\right)^{H}$. Let $\mathcal{W} \subset N_{K}\left(\mathfrak{a}_{\mathrm{q}}\right)$ be a fixed set of elements such that $w \mapsto H w P$ parametrizes the open $H \times P$ orbits on $G$ (it is known (see [59] or [49]) that any set of representatives for the double quotient $N_{K \cap H}\left(\mathfrak{a}_{\mathrm{q}}\right) \backslash N_{K}\left(\mathfrak{a}_{\mathrm{q}}\right) / Z_{K}\left(\mathfrak{a}_{\mathrm{q}}\right)$ can be used as $\mathcal{W}$ - in particular, $\mathcal{W}$ is finite). Viewing an element $f \in \mathcal{H}_{\xi, \lambda}^{-\infty}$ as an $\mathcal{H}_{\xi}$ valued distribution on $G$, satisfying appropriate conditions of homogeneity for the right action of $P$, it is easily seen that if $f$ is $H$-invariant then $f$ must restrict to a smooth function on each open $H \times P$ orbit. Hence it makes sense to evaluate $f$ in the elements of $\mathcal{W}$, and in fact its restriction to the open orbit $H w P$ will be uniquely determined from the value at $w$. We denote this value by $\operatorname{ev}_{w}(f)$. It is easily seen that ev $w$ maps $\mathcal{V}_{\xi, \lambda}$ into the space $\mathcal{H}_{\xi}^{w^{-1}(M \cap H) w}$ of $w^{-1}(M \cap H) w$-fixed elements in $\mathcal{H}_{\xi}$ (note that $w^{-1} M w=M$, but $w^{-1} H w$ may differ from $H$ ). Let $V(\xi)$ denote the formal direct sum

$$
V(\xi)=\bigoplus_{w \in \mathcal{W}} \mathcal{H}_{\xi}^{w^{-1}(M \cap H) w}
$$

provided with the direct sum inner product (thus, by definition the summands are mutually orthogonal, even though this may not be the case in $\mathcal{H}_{\xi}$ ). Furthermore, let

$$
\mathrm{ev}: \mathcal{V}_{\xi, \lambda} \rightarrow V(\xi)
$$

denote the direct sum of the maps $\mathrm{ev}_{w}$. The construction of the induced representations $\pi_{\xi, \lambda}$ and of the map ev makes sense for $\lambda \in \mathfrak{a}_{\mathbf{q}}^{*} \mathbb{C}$, the complex linear dual of $\mathfrak{a}_{\mathrm{q}}$ (though the representations need not be unitary for $\lambda$ outside $i \mathfrak{a}_{\mathrm{q}}^{*}$ ). We now have

Theorem 4, [3]. The map ev is bijective for generic $\lambda \in \mathfrak{a}_{\mathrm{q}}^{*}$.

(In this context 'generic' means outside a countable union of complex hyperplanes). For generic $\lambda$, let

$$
j(\xi, \lambda): V(\xi) \rightarrow \mathcal{V}_{\xi, \lambda}
$$

be the inverse of ev, then by definition we have for $\eta \in V(\xi)$ that the restriction of the distribution $j(\xi, \lambda)(\eta)$ to the open $H \times P$ orbit $H w P, w \in \mathcal{W}$, is the smooth $\mathcal{H}_{\xi}$-valued function given by

$$
j(\xi, \lambda)(\eta)(h w \operatorname{man})=a^{-\lambda-\rho} \xi\left(m^{-1}\right) \eta_{w} .
$$

(Here $\eta_{w}$ denotes the $w$-component of $\eta$, viewed as an element of $\mathcal{H}_{\xi} \cdot$ ) Notice that if $G / H$ is a Riemannian symmetric space, so that $H=K$, then we have $G=H P$ by 
the Iwasawa decomposition. Hence we can take $\mathcal{W}=\{e\}$, and since $M \subset K=H$ we have $V(\xi)=\{0\}$ unless $\xi$ is the trivial representation $\mathbf{1}$, in which case $V(\mathbf{1})=\mathbb{C}$. Then $j(\mathbf{1}, \lambda)$ is completely determined by (12); in fact we have

$$
j(\mathbf{1}, \lambda)(x)=e^{-(\lambda+\rho) H(x)},
$$

where $H: G \rightarrow \mathfrak{a}$ is the Iwasawa projection (since $V(\mathbf{1})=\mathbb{C}$ we can omit $\eta$ ). Thus the kernel $p_{\lambda}(x, k)=j(\mathbf{1}, \lambda)\left(x^{-1} k\right)$ on $G / K \times K$ is the generalized Poisson kernel. For general $G / H$ we can supplement (12) as follows: If $\operatorname{Re}\langle\lambda+\rho, \alpha\rangle<0$ for all $\alpha$ in the set $\Sigma^{+}$of positive roots (the $\mathfrak{a}-\operatorname{roots}$ of $\mathfrak{n}=\operatorname{Lie}(N)$ ), then $j(\xi, \lambda)(\eta)$ is the continuous function on $G$ given by (12) on $H w P$ for all $w \in \mathcal{W}$ and vanishing on the complement of these sets (the condition on $\lambda$ ensures the continuity). For elements $\lambda$ outside the above region the distribution $j(\xi, \lambda)$ can be obtained from the above by meromorphic continuation. (See [56], [53], [3]. These results have been generalized to other principal series representations in [21], [24].)

Having constructed the $H$-invariant distribution vectors $j(\xi, \lambda) \eta$ as above we can now attempt to write down a Fourier transform for the principal series. For $f \in C_{c}^{\infty}(G / H)$ we consider the map

$$
(\xi, \lambda) \mapsto f^{\wedge}\left(\pi_{\xi, \lambda}\right) j(\xi, \lambda)=\pi_{\xi, \lambda}(f) j(\xi, \lambda) \in \mathcal{H}_{\xi, \lambda}^{\infty} \otimes V(\xi)^{*}
$$

In the Riemannian case this is exactly the Fourier transform, as defined by Helgason (see [42]). However when $G / H$ is not Riemannian a new phenomenon may occur: by the above definitions (13) is a meromorphic function in $\lambda$, which may have singularities on the set $i \mathfrak{a}_{q}^{*}$ of interest for the Plancherel decomposition, and thus it may not make sense for some singular $\lambda \in i \mathfrak{a}_{\mathrm{q}}^{*}$. This unpleasantness is overcome by a suitable normalization of $j(\xi, \lambda)$, which removes the singularities. The normalization is carried out by means of the standard intertwining operators $A(\bar{P}, P, \xi, \lambda)$ from $\pi_{P, \xi, \lambda}$ to $\pi_{\bar{P}, \xi, \lambda}$, where $\bar{P}$ is the parabolic subgroup opposite to $P$. Let

$$
j^{\circ}(\xi, \lambda)=A(\bar{P}, P, \xi, \lambda)^{-1} j(\bar{P}, \xi, \lambda),
$$

where $j(\bar{P}, \xi, \lambda)$ is constructed as $j(\xi, \lambda)$ above, but with $P$ replaced by $\bar{P}$. Since the intertwining operator $A(\bar{P}, P, \xi, \lambda)$ is bijective for generic $\lambda$, it follows that

$$
j^{\circ}(\xi, \lambda): V(\xi) \rightarrow \mathcal{V}_{\xi, \lambda}
$$

is again a bijection, for generic $\lambda$. Moreover, we now have

Theorem 5, [11]. The meromorphic function $\lambda \mapsto j^{\circ}(\xi, \lambda)$ is regular on $i \mathfrak{a}_{\mathrm{q}}^{*}$.

We can now define the Fourier transform $f \mapsto f^{\wedge}$ for the principal series properly by (13), but with $j$ replaced by $j^{\circ}$ :

$$
f^{\wedge}(\xi, \lambda)=\pi_{\xi, \lambda}(f) j^{\circ}(\xi, \lambda) \in \mathcal{H}_{\xi, \lambda}^{\infty} \otimes V(\xi)^{*} .
$$


Notice that when $G / H$ is Riemannian the normalization makes our Fourier transform different from that of Helgason - in this case the normalization amounts to a division by Harish-Chandra's $c$-function $\mathbf{c}(\lambda)$. See $[10]$ for the determination of $j^{\circ}$ in the group case.

We can now give the solution to Problem (b) for this part of $L^{2}(G / H)$ : We take $\mathcal{V}_{\xi, \lambda}^{o}=\mathcal{V}_{\xi, \lambda}$, and give it the Hilbert space structure that makes $j^{\circ}(\xi, \lambda)$ an isometry. The solution to Problem (c) is as follows. Let $\mathcal{H}$ be the Hilbert space given by

$$
\mathcal{H}=\int_{\xi, \lambda}^{\oplus} \mathcal{H}_{\xi, \lambda} \otimes V(\xi)^{*} d \mu(\xi, \lambda)
$$

with the measure $d \mu(\xi, \lambda)=\operatorname{dim}(\xi) d \lambda$, where $d \lambda$ is Lebesgue measure on $i \mathfrak{a}_{\mathrm{q}}^{*}$ (suitably normalized). Here $\xi$ runs over $M_{\text {fu }}^{\wedge}$ (notice however that some of them may disappear because $V(\xi)$ is trivial), and $\lambda$ runs over an open chamber $i \mathfrak{a}_{\mathrm{q}}^{*+}$ in $i \mathfrak{a}_{\mathrm{q}}^{*}$ for the Weyl group $W_{\mathrm{q}}=N_{K}\left(\mathfrak{a}_{\mathrm{q}}\right) / Z_{K}\left(\mathfrak{a}_{\mathrm{q}}\right)$.

Theorem 6, [13]. Let $f \in C_{c}^{\infty}(G / H)$. Then $f^{\wedge} \in \mathcal{H}$ and $\left\|f^{\wedge}\right\| \leq\|f\|_{2}$. Moreover, the map $f \mapsto f^{\wedge}$ extends to an equivariant partial isometry $\mathfrak{F}$ of $L^{2}(G / H)$ onto $\mathcal{H}$. In particular, the multiplicity of $\pi_{\xi, \lambda}$ is $m_{\xi}=\operatorname{dim} V(\xi)$ for almost all $\lambda$.

We define the most continuous part $L_{\mathrm{mc}}^{2}(G / H)$ of $L^{2}(G / H)$ as the orthocomplement of the kernel of $\mathfrak{F}$. Then $\mathfrak{F}$ restricts to an isometry of this space onto $\mathcal{H}$. In [13] it is shown that $L_{\mathrm{mc}}^{2}(G / H)$ is 'large' in $L^{2}(G / H)$ in a certain sense - in particular its orthocomplement (the kernel of $\mathfrak{F}$ ) has trivial intersection with $C_{c}^{\infty}(G / H)$ (thus $f \mapsto f^{\wedge}$ is injective, even though the extension $\mathfrak{F}$ need not be). Moreover, if $G / H$ has split rank one, that is if $\operatorname{dim} \mathfrak{a}_{\mathrm{q}}=1$, then there are at most two conjugacy classes of Cartan subspaces, and hence one expects from the analogy with the group case as mentioned earlier that only the corresponding two 'series' of representations will be present. Indeed this is the case; it is shown in [13] that the kernel of $\mathfrak{F}$ decomposes discretely when the split rank is one. Thus, in this case the Plancherel decomposition of $L^{2}(G / H)$ can be determined from Theorem 6 together with the description of the discrete series (see Section 4 above), except for the explicit determination of the Hilbert space structure on $\mathcal{V}_{\pi}^{o}$ for the discrete series representations $\pi$.

On the other hand, when $G / H$ is Riemannian then $\mathfrak{F}$ is injective and Theorem 6 gives the complete Plancherel decomposition of $L^{2}(G / H)$ (in the formulation of Harish-Chandra and Helgason the Plancherel measure is $|\mathbf{c}(\lambda)|^{-2} d \lambda$, but here the factor $|\mathbf{c}(\lambda)|^{-2}$ disappears because of the normalization of $j^{\circ}$ ).

A further discussion of the multiplicities $m_{\pi}$ can be found in [10].

\subsection{The SPHERICAL Fourier TRANSForm}

The isomorphism of (14) onto $L_{\mathrm{mc}}^{2}(G / H)$ (the 'inverse Fourier transform') can be given more explicitly when one restricts to $K$-finite functions. In this subsection we 
shall discuss this restriction, which happens to be crucial in the proofs of Theorems 5 and 6.

5.2.1. Eisenstein integrals. Let $\left(\mu, V_{\mu}\right)$ be a fixed, irreducible unitary representation of $K$. Taking $\mu$-components in (14) we have

$$
\mathcal{H}^{\mu}=\int_{\xi, \lambda}^{\oplus} \mathcal{H}_{\xi, \lambda}^{\mu} \otimes V(\xi)^{*} d \mu(\xi, \lambda) .
$$

Moreover, by Frobenius reciprocity we have

$$
\mathcal{H}_{\xi, \lambda}^{\mu} \simeq \operatorname{Hom}_{M \cap K}\left(V_{\mu}, \mathcal{H}_{\xi}\right) \otimes V_{\mu}
$$

as $K$-modules (where $K$ acts on the second component in the tensor product), for all $\xi \in M_{\mathrm{fu}}^{\wedge}, \lambda \in \mathfrak{a}_{\mathrm{q}}^{*} \mathbb{C}$. Note that since each representation $\xi \in M_{\mathrm{fu}}^{\wedge}$ is trivial on the noncompact part of $M$, we have that $\left.\xi\right|_{M \cap K}$ is irreducible, and that $\operatorname{Hom}_{M \cap K}\left(V_{\mu}, \mathcal{H}_{\xi}\right)$ is non-trivial if and only if this restriction occurs as a subrepresentation of $\left.\mu\right|_{M \cap K}$. We use the notation $\xi \uparrow \mu$ to indicate this occurrence; it happens only for finitely many $\xi$. Thus by taking $K$-types the integral over $\xi$ in (15) becomes a finite sum, hence more manageable. In analogy with the earlier definition of the space $V(\xi)$ we now define the space $\mathcal{V}(\mu)$ to be the formal direct sum

$$
\mathcal{V}(\mu)=\bigoplus_{w \in \mathcal{W}} V_{\mu}^{w^{-1}(K \cap M \cap H) w} .
$$

It is easily seen from the above that

$$
\mathcal{V}(\mu) \simeq \bigoplus_{\xi \uparrow \mu} \operatorname{Hom}_{M \cap K}\left(\mathcal{H}_{\xi}, V_{\mu}\right) \otimes V(\xi)
$$

Hence in view of (16) we have

$$
\mathcal{V}(\mu)^{*} \otimes V_{\mu} \simeq \bigoplus_{\xi \mid \mu} \mathcal{H}_{\xi, \lambda}^{\mu} \otimes V(\xi)^{*}
$$

for all $\lambda \in \mathfrak{a}_{\mathbf{q}}^{*} \mathbb{C}$. From (15) and (18) we finally obtain

$$
\mathcal{H}^{\mu} \simeq \int_{\lambda}^{\oplus} \mathcal{V}(\mu)^{*} \otimes V_{\mu} d \lambda \simeq L^{2}\left(i \mathfrak{a}_{\mathrm{q}}^{*+}\right) \otimes \mathcal{V}(\mu)^{*} \otimes V_{\mu} .
$$

This isomorphism indicates that the Fourier transform, when restricted to $K$-finite functions of type $\mu$, can be considered as a map into the $\mathcal{V}(\mu)^{*} \otimes V_{\mu}$-valued functions on $i \mathfrak{a}_{\mathrm{q}}^{*}$.

Instead of working with $K$-finite scalar-valued functions on $G / H$, it is convenient to consider ' $\mu$-spherical' functions $f$ on $G / H$, that is, $V_{\mu}$-valued functions satisfying

$$
f(k x)=\mu(k) f(x), \quad k \in K, x \in G / H .
$$


Let $L^{2}(G / H ; \mu)$ denote the space of square integrable such functions, then by contraction we have a $K$-equivariant isomorphism

$$
\gamma_{\mu}: L^{2}\left(G / H ; \mu^{\vee}\right) \otimes V_{\mu} \stackrel{\sim}{\longrightarrow} L^{2}(G / H)^{\mu}
$$

(Again $K$ acts on the second component in the tensor product. The map $\operatorname{dim}(\mu) \gamma_{\mu}$ is an isometry.) Notice that when passing from $K$-finite functions to spherical functions one must also pass from $\mu$ to its contragradient $\mu^{\vee}$. Since $\mathcal{V}(\mu)^{*}=\mathcal{V}\left(\mu^{\vee}\right)$ we are led to the search, for each $\mu$, of a Fourier transform, which is a partial isometry of $L^{2}(G / H ; \mu)$ onto $L^{2}\left(i \mathfrak{a}_{\mathrm{q}}^{*+}\right) \otimes \mathcal{V}(\mu)$. Going through the above isomorphisms in detail, we are led to the following construction culminating in (26), which essentially is the 'projection' of the construction of $f \mapsto f^{\wedge}$ to functions of type $\mu$.

For $\psi \in \mathcal{V}(\mu)$ and $\lambda \in \mathfrak{a}_{\mathrm{q}}^{*} \mathbb{C}$ with $\operatorname{Re}\langle\lambda+\rho, \alpha\rangle<0$ for all $\alpha \in \Sigma^{+}$, let $\tilde{\psi}_{\lambda}$ be the $V_{\mu}$-valued function on $G$ defined by

$$
\tilde{\psi}_{\lambda}(x)= \begin{cases}a^{-\lambda-\rho} \mu\left(m^{-1}\right) \psi_{w} & \text { if } x=h w \operatorname{man} \in H w(M \cap K) A N, w \in \mathcal{W}, \\ 0 & \text { if } x \notin \cup_{w \in \mathcal{W}} H w P,\end{cases}
$$

where $\psi_{w}$ denotes the $w$-component of $\psi$. (It is to be noted that $M=w^{-1}(M \cap$ $H) w(M \cap K)$, and hence $H w(M \cap K) A N=H w M A N$.) It can be shown that $\tilde{\psi}_{\lambda}$ is continuous as a function of $x$, and has a distribution-valued meromorphic continuation in $\lambda \in \mathfrak{a}_{\mathrm{q}}^{*}$. Let $E_{\mu}(\psi, \lambda)$ be the $\mu$-spherical function on $G / H$ defined by

$$
E_{\mu}(\psi, \lambda)(x)=\int_{K} \mu(k) \tilde{\psi}_{\lambda}\left(x^{-1} k\right) d k .
$$

It can be seen that the vector components of $E_{\mu}(\psi, \lambda)$ are linear combinations of generalized matrix coefficients formed by the $j(\xi, \lambda) \eta,\left(\eta \in V(\xi), \xi \uparrow \mu^{\vee}\right)$, with $K$-finite vectors of type $\mu$; in particular, $E_{\mu}(\psi, \lambda)$ is a smooth function on $G / H$, even when $\tilde{\psi}_{\lambda}$ is only a distribution. We call these functions Eisenstein integrals for $G / H$. When $G / H$ is Riemannian and $\mu$ is the trivial $K$-type $\mathbf{1}$, the construction produces the spherical functions

$$
\varphi_{\lambda}(x)=\int_{K} e^{-(\lambda+\rho) H\left(x^{-1} k\right)} d k
$$

and for other $K$-types we get the generalized spherical functions of [44]. In the group case the Eisenstein integrals defined in this manner coincide, up to normalization, with Harish-Chandra's Eisenstein integrals associated to the minimal parabolic subgroup.

The spherical functions are eigenfunctions for the invariant differential operators on $G / K-$ in analogy we have

$$
D E_{\mu}(\psi, \lambda)=E_{\mu}\left(\chi_{\mu}(D, \lambda) \psi, \lambda\right)
$$


for all $D \in \mathbb{D}(G / H)$. Here $\chi_{\mu}(D)$ is an $\operatorname{End}(\mathcal{V}(\mu))$-valued polynomial in $\lambda$. Just as it is the case for the spherical functions, one can derive an asymptotic expansion from this 'eigenequation'. Here we have to recall the ' $K A H$ '-decomposition of $G$,

$$
G=\mathrm{cl} \bigcup_{w \in \mathcal{W}} K A_{\mathrm{q}}^{+} w^{-1} H
$$

where $A_{q}^{+}$is the exponential of the positive chamber in $\mathfrak{a}_{\mathrm{q}}$ corresponding to $\Sigma^{+}$, and where the union inside the closure operator $\mathrm{cl}$ is disjoint. Since the Eisenstein integrals are $K$-spherical, we have to consider their behavior on $A_{\mathrm{q}}^{+} w^{-1}$, for all $w \in$ $\mathcal{W}$. Notice that when $G / H$ is Riemannian there is only one 'direction' to control, since the $K A H$-decomposition then specializes to the Cartan decomposition $G=$ cl $K A^{+} K$. The expansion is essentially as follows (see [4]):

$$
E_{\mu}(\psi, \lambda)\left(a w^{-1}\right)=\sum_{s \in W_{\mathrm{q}}} a^{s \lambda-\rho}[C(s, \lambda) \psi]_{w}+\text { lower order terms in } a,
$$

for $a \in A_{\mathrm{q}}^{+}, w \in \mathcal{W}$, where $W_{\mathrm{q}}$ is as defined above Theorem 6 , and the ' $c$-function' $\lambda \mapsto C(s, \lambda)$ is a meromorphic function on $\mathfrak{a}_{\mathbf{q}}^{*} \mathbb{C}$ with values in $\operatorname{End}(\mathcal{V}(\mu)$ ) (it follows easily from the $\mu$-sphericality that we have $E_{\mu}(\psi, \lambda)\left(a w^{-1}\right) \in V_{\mu}^{w^{-1}(K \cap M \cap H) w}$ for $a \in A_{\mathrm{q}}$ ). The expansion converges for $a \in A_{\mathrm{q}}^{+}$; the 'lower order terms' involve powers of the form $a^{s \lambda-\rho-\nu}$ where $\nu$ is a sum of positive roots.

The expression (24) is analyzed in [12], where it is shown that it takes the form

$$
E_{\mu}(\psi, \lambda)\left(a w^{-1}\right)=\sum_{s \in W_{\mathrm{q}}} \Phi_{w}(s \lambda, a)[C(s, \lambda) \psi]_{w}
$$

for each $w \in \mathcal{W}$, where $\Phi_{w}(\lambda, \cdot) \in \operatorname{End}\left(V_{\mu}^{w^{-1}(K \cap H \cap M) w}\right)$ is given on $A_{\mathrm{q}}^{+}$by a converging power series with $a^{\lambda-\rho}$ as its leading term.

5.2.2. The Fourier transform. It would now be natural to define the Fourier transform $\mathcal{F}_{\mu} f$ of a function $f \in C_{c}^{\infty}(G / H ; \mu)$, the space of compactly supported and smooth $\mu$-spherical functions on $G / H$, as the $\mathcal{V}(\mu)$-valued function $\varphi$ on $\mathfrak{a}_{\mathrm{q}}^{*} \mathbb{C}$ given by

$$
\langle\varphi(\lambda) \mid \psi\rangle=\int_{G / H}\left\langle f(x) \mid E_{\mu}(\psi,-\bar{\lambda})(x)\right\rangle d x, \quad \psi \in \mathcal{V}(\mu)
$$

where the inner products $\langle\cdot \mid \cdot\rangle$ are the sesquilinear Hilbert space inner products on $\mathcal{V}(\mu)$ and $V_{\mu}$, respectively. Via the isomorphisms in (19) and (20) this would essentially correspond to the Fourier transform in (13). However, as with $j(\xi, \lambda)$ we have the problem that $E_{\mu}(\psi, \lambda)$, which is meromorphic in $\lambda$, may have singularities on $i \mathfrak{a}_{\mathrm{q}}^{*}$. Again we have to carry out a normalization: the normalized Eisenstein integral is defined by

$$
E_{\mu}^{\circ}(\psi, \lambda)=E_{\mu}\left(C(1, \lambda)^{-1} \psi, \lambda\right)
$$


In other words, the Eisenstein integral is normalized by its asymptotics, so that we have $E_{\mu}^{\circ}(\psi, \lambda)\left(a w^{-1}\right) \sim a^{\lambda-\rho} \psi_{w}$ for $a \in A_{\mathrm{q}}^{+}, w \in \mathcal{W}$ and $\operatorname{Re} \lambda$ strictly dominant. It can be shown that this normalization corresponds to the one on $j(\xi, \lambda)$, in the sense that the vector components of $E_{\mu}^{\circ}(\psi, \lambda)$ are linear combinations of matrix coefficients formed by the $j^{\circ}(\xi, \lambda) \eta,\left(\eta \in V(\xi), \xi \uparrow \mu^{\vee}\right)$, with $K$-finite vectors of type $\mu$. Moreover, it can be shown that the statement of Theorem 5 is equivalent with the following ' $K$-finite version':

Theorem 7, [11]. The meromorphic function $\lambda \mapsto E_{\mu}^{\circ}(\psi, \lambda)$ is regular on $i \mathfrak{a}_{\mathrm{q}}^{*}$, for every $\mu \in K^{\wedge}$ and $\psi \in \mathcal{V}(\mu)$.

A proof of Theorem 7, different from the original proof in [11] and valid for the generalized principal series as well, is given in [7]. With the result of Theorem 7 in mind we define the $\mu$-spherical Fourier transform $\mathcal{F}_{\mu} f$ as above, but with $E_{\mu}$ replaced by $E_{\mu}^{\circ}$, that is, by

$$
\left\langle\mathcal{F}_{\mu} f(\lambda) \mid \psi\right\rangle=\int_{G / H}\left\langle f(x) \mid E_{\mu}^{\circ}(\psi,-\bar{\lambda})(x)\right\rangle d x, \quad \psi \in \mathcal{V}(\mu) .
$$

Then $\mathcal{F}_{\mu} f$ corresponds to $f^{\wedge}$ via the isomorphisms in (18) and (20).

When $G / H$ is Riemannian and $\mu=\mathbf{1}$, the normalization again amounts to division by $\mathbf{c}(\lambda)$, and thus $\mathcal{F}_{\mu} f$ is in this case related to the spherical Fourier transform of $f$ as follows:

$$
\mathcal{F}_{\mu} f(\lambda)=\mathbf{c}(-\lambda)^{-1} \int_{G / K} f(x) \varphi_{-\lambda}(x) d x
$$

where $\varphi_{\lambda}$ is the elementary spherical function in (21). If $G / H$ is Riemannian and $\mu$ is non-trivial there is a similar relation, also involving $\mathbf{c}(\lambda)^{-1}$, to the Fourier transform in [44].

Let $C^{\circ}(s, \lambda)=C(s, \lambda) C(1, \lambda)^{-1}$, then we have from $(24)-(25)$

$$
\begin{aligned}
E_{\mu}^{\circ}(\psi, \lambda)\left(a w^{-1}\right) & =\sum_{s \in W_{\mathrm{q}}} a^{s \lambda-\rho}\left[C^{\circ}(s, \lambda) \psi\right]_{w}+\text { lower order terms in } a \\
& =\sum_{s \in W_{\mathrm{q}}} \Phi_{w}(s \lambda, a)\left[C^{\circ}(s, \lambda) \psi\right]_{w} .
\end{aligned}
$$

The following theorem generalizes results of Helgason and Harish-Chandra for the Riemannian case and the group case, respectively (see [43, Thm. 6.6], [39, Lemma 17.6], the Maass-Selberg relations).

Theorem 8, [4], [5]. For every $s \in W_{\mathrm{q}}$ we have the following identity of meromorphic functions:

$$
C^{\circ}(s, \lambda) C^{\circ}(s,-\bar{\lambda})^{*}=I_{\mathcal{V}(\mu)} \quad\left(\lambda \in \mathfrak{a}_{\mathbf{q}}^{*} \mathbb{C}\right)
$$


In particular, for $\lambda \in i \mathfrak{a}_{\mathrm{q}}^{*}$, the endomorphism $C^{\circ}(s, \lambda)$ of $\mathcal{V}(\mu)$ is unitary.

Notice that by Riemann's boundedness theorem it follows from the above result that the meromorphic function $\lambda \mapsto C^{\circ}(s, \lambda)$ has no singularities on $i \mathfrak{a}_{\mathrm{q}}^{*}$. Therefore the possible singularities of $E_{\mu}^{\circ}(\psi, \lambda)$ must occur in the lower order terms of $(27)$. This observation plays a crucial role in the proof of Theorem 7 .

On $G / K$ the spherical functions satisfy the functional equation $\varphi_{s \lambda}=\varphi_{\lambda}$, for all $s \in W_{\mathrm{q}}$. The analog for the normalized Eisenstein integral on $G / H$ is

$$
E_{\mu}^{\circ}\left(C^{\circ}(s, \lambda) \psi, s \lambda\right)=E_{\mu}^{\circ}(\psi, \lambda)
$$

(see [4, Prop. 16.4]. For the group case, see also [39, Lemma 17.2]).

Though $E_{\mu}^{\circ}(\psi, \lambda)$ by Theorem 7 is regular on $i \mathfrak{a}_{q}^{*}$, it will in general have singularities elsewhere on $\mathfrak{a}_{\mathfrak{q}}^{*}$. It is remarkable, though, that in a certain direction only finitely many singularities occur. To be more precise, one has the following. Let

$$
\left(\mathfrak{a}_{\mathrm{q}}^{*}\right)_{+}=\left\{\lambda \in \mathfrak{a}_{\mathrm{q}}^{*} \mid \operatorname{Re}\langle\lambda, \alpha\rangle \geq 0, \alpha \in \Sigma^{+}\right\},
$$

and put $\left(\mathfrak{a}_{\mathbf{q} \mathbb{C}}^{*}\right)_{-}=-\left(\mathfrak{a}_{\mathbf{q}}^{*}\right)_{+}$.

Theorem 9, [4]. There exists a polynomial $\pi^{\prime}$ on $\mathfrak{a}_{\mathrm{q}}^{*} \mathbb{C}$, which is a product of linear factors of the form $\lambda \mapsto\langle\lambda, \alpha\rangle+$ constant, with $\alpha$ a root, such that $\pi^{\prime}(\lambda) E_{\mu}^{\circ}(\psi, \lambda)$ is holomorphic on a neighborhood of $\left(\mathfrak{a}_{\mathrm{q}}^{*} \mathbb{C}\right)_{+}$.

Notice that $\pi^{\prime}$ depends on the $K$-type $\mu$. Notice also that when $G / H$ is Riemannian we actually have that $E_{\mu}^{\circ}(\psi, \lambda)$ itself is holomorphic on $\left(\mathfrak{a}_{\mathrm{q}}^{*}\right)_{+} \cdot$ Indeed, the spherical functions are everywhere holomorphic, and the normalizing divisor $\mathbf{c}(\lambda)$ has no zeros on this set. Thus, for this case one can take $\pi^{\prime}=1$.

It follows from Theorem 9 and (26) that if we put

$$
\pi(\lambda)=\overline{\pi^{\prime}(-\bar{\lambda})}
$$

then $\lambda \mapsto \pi(\lambda) \mathcal{F}_{\mu} f(\lambda)$ is holomorphic on a neighborhood of $\left(\mathfrak{a}_{\mathrm{q}}^{*} \mathbb{C}\right)_{-}$.

5.2.3. Wave packets. For the $\mu$-spherical Fourier transform a "partial inversion formula' is given in [13] as follows. For a $\mathcal{V}(\mu)$-valued function $\varphi$ on $i \mathfrak{a}_{\mathrm{q}}^{*}$ of suitable decay one can form a 'wave packet', which is the superposition of normalized Eisenstein integrals, with amplitudes given by $\varphi$, that is

$$
\mathcal{J}_{\mu} \varphi(x)=\int_{i \mathfrak{a}_{\mathrm{q}}^{*}} E_{\mu}^{\circ}(\varphi(\lambda), \lambda)(x) d \lambda \quad(x \in G / H) .
$$

It is easily seen that the transform $\mathcal{J}_{\mu}$ is the transpose of $\mathcal{F}_{\mu}$. For Euclidean Fourier transform (and more generally for the spherical Fourier transform on a Riemannian symmetric space) this transform is also the inverse of $\mathcal{F}_{\mu}$; the inversion formula states that $\mathcal{J}_{\mu} \mathcal{F}_{\mu}$ is the identity operator (when measures are suitably normalized). In the non-Riemannian generality of $G / H$ this cannot be expected, because of the possible presence of discrete series. However we do have 
Theorem 10, [13]. There exists an invariant differential operator $D$ (depending on $\mu$ ) on $G / H$ satisfying the following:

(i) As an operator on $C_{c}^{\infty}(G / H), D$ is injective and symmetric.

(ii) $\mathcal{J}_{\mu} \mathcal{F}_{\mu} f=f$ for all $f \in D\left(C_{c}^{\infty}(G / H ; \mu)\right)$.

From (22) one can derive that $\mathcal{J}_{\mu} \mathcal{F}_{\mu} D=\mathcal{J}_{\mu} \chi_{\mu}(D) \mathcal{F}_{\mu}=D \mathcal{J}_{\mu} \mathcal{F}_{\mu}$. Hence it follows from (ii) that $D\left(\mathcal{J}_{\mu} \mathcal{F}_{\mu} f-f\right)=0$ for all $f \in C_{c}^{\infty}(G / H ; \mu)$. Nevertheless, one cannot then conclude from (i) that in fact $\mathcal{J}_{\mu} \mathcal{F}_{\mu} f=f$ because $\mathcal{J}_{\mu} \mathcal{F}_{\mu} f$ is not compactly supported in general. The presence of $D$ is important, for example it annihilates all the discrete series in $L^{2}(G / H ; \mu)$.

The proof of Theorem 10 is very much inspired by Rosenberg's proof (see [58] or $[46, \mathrm{Ch} . \mathrm{IV}, \S 7])$ of the inversion formula for the spherical Fourier transform on $G / K$ (in which case one can take $D=1$ ). A key step in both proofs is the use of a 'shift argument', originally used by Helgason for the proof of the Paley-Wiener theorem, where the integration in $\mathcal{J}_{\mu}$ (after use of (27)) is moved away from $i \mathfrak{a}_{\mathrm{q}}^{*}$ in the direction of $\left(\mathfrak{a}_{\mathbf{q} \mathbb{C}}^{*}\right)_{-}$, using Cauchy's theorem. It can be seen that one only meets a finite number of singular hyperplanes in this shift. The purpose of the operator $D$ is to remove these singularities (among other things this means that $\pi$ should be a divisor in $\left.\chi_{\mu}(D)\right)$, so that no residues are present. The shift allows one to conclude that $\mathcal{J}_{\mu} \mathcal{F}_{\mu} D f$ is compactly supported whenever $f$ is, which is an important step in the proof of the theorem.

Theorem 10 is crucial in the proof of Theorem 6. Via the isomorphism (20) one obtains with $\mathcal{J}_{\mu^{\vee}}$ an explicit formula for the restriction to $\mathcal{H}^{\mu}$ of the isomorphism of $\mathcal{H}$ onto $L_{\mathrm{mc}}^{2}(G / H)$.

\section{The Plancherel formula for $L^{2}(G / H)$. The intermediate series}

In a more recent development than what was described above, both the Plancherel formula for the full space $L^{2}(G / H)$ and the Paley-Wiener theorem have been obtained. Both of these results were announced in the seminar at the Mittag-Leffler Institute in November, 1995.

The Plancherel theorem was announced by Delorme; the proof has appeared in [29]. (In 1986 Oshima announced that he had obtained a Plancherel formula, see [54, p. 604], but the details have not appeared).

The Paley-Wiener theorem was announced by the first and last named author of the present paper. They also announced that their proof implies the Plancherel formula for spaces with one conjugacy class of Cartan subspaces, and that in general their proof implies the Plancherel formula under the hypothesis that the identity of Theorem 8 (the Maass-Selberg relations) is valid for generalized Eisenstein integrals (see below). The validity of this hypothesis, which also plays a main role in Delorme's work, has been established by Carmona and Delorme in [25]. The details of the work of van den Ban and Schlichtkrull will appear in [16]. 
The theory of Eisenstein integrals that was developed in the previous section for the most continuous part of $L^{2}(G / H)$ can be generalized to the intermediate series as well. This has been done in a series of papers by Delorme and others, [21], [24], [23], [7], [27], [28], [25]. In the above we referred already to the generalization (in [25]) of the identity in Theorem 8 (the Maass-Selberg relations) to these intermediate series. The proof is based on the method of truncation, which was introduced in this context by Delorme in [28]. As a consequence of the generalization of Theorem 8, the regularity in Theorem 7 is extended (also in [25]) to the (generalized) Eisenstein integrals corresponding to the intermediate series. These results are of significant importance in both of the mentioned approaches to the Plancherel formula. Another important ingredient in [29] (but not in [16]) is an a priori characterization of the support of the Plancherel measure (cf [24, Appendix C]), which in turn is derived from a result of Bernstein [18].

In [16] the Plancherel formula is derived from an inversion formula for the Fourier transform $\mathcal{F}_{\mu}$ that was defined on $C_{c}^{\infty}(G / H ; \mu)$ in (26). This inversion formula is based on the 'shift argument' that was described after Theorem 10. Without the presence of the operator $D$ one obtains by this shift an expression involving generalized residues. It is these residues that give rise to the intermediate and the discrete series. At this point the method resembles (and was, in fact, inspired by) that of Langlands (see [51]), Arthur [1] and of Heckman and Opdam [40]. A selfcontained theory for the involved residue calculus for root systems is developed in [14].

To be somewhat more specific, let $(G / H)_{+} \subset G / H$ be the dense open subset

$$
(G / H)_{+}=\bigcup_{w \in \mathcal{W}} K A_{\mathrm{q}}^{+} w^{-1} H
$$

(see $(23))$, and define a $\operatorname{Hom}\left(\mathcal{V}(\mu), V_{\mu}\right)$-valued function $E_{+}(\lambda, \cdot)$ on $(G / H)_{+}$by

$$
E_{+}\left(\lambda, k a w^{-1}\right) \psi=\mu(k) \Phi_{w}(\lambda, a) \psi_{w}
$$

(see (25)) for $\lambda \in \mathfrak{a}_{\mathrm{q}}^{*} \mathbb{C}$ generic, $k \in K, a \in A_{\mathrm{q}}^{+}, w \in \mathcal{W}$ and $\psi \in \mathcal{V}(\mu)$. Then (27) takes the form

$$
E_{\mu}^{\circ}(\psi, \lambda)(x)=\sum_{s \in W_{\mathrm{q}}} E_{+}(\lambda, x) C^{\circ}(s, \lambda) \psi, \quad\left(x \in(G / H)_{+}\right) .
$$

We define, for $f \in C_{c}^{\infty}(G / H ; \mu), x \in(G / H)_{+}$and $\eta \in \mathfrak{a}_{\mathrm{q}}^{*}$ generic

$$
\mathcal{T}_{\mu}^{\eta} \mathcal{F}_{\mu} f(x)=\left|W_{\mathrm{q}}\right| \int_{\eta+i \mathfrak{a}_{\mathrm{q}}^{*}} E_{+}(\lambda, x) \mathcal{F}_{\mu} f(\lambda) d \lambda \in V_{\mu}
$$

it can be shown that this integral converges and defines a smooth function on $(G / H)_{+}$. The previously mentioned shift argument involves two steps. The first 
step is the identification of the wave packet $\mathcal{J}_{\mu} \mathcal{F}_{\mu} f$ with $\mathcal{T}_{\mu}^{\eta} \mathcal{F}_{\mu} f$ for $\eta=0$ (or, if this is a singular value, with a certain limit). This is done simply by insertion of (31) in the integral (30) that defines the wave packet. The second step is the actual shift. In the integral (32) $\eta$ is shifted from 0 towards infinity in the antidominant direction. During this shift a finite number of singular hyperplanes is passed, and some generalized residues are created. For $\eta$ sufficiently antidominant all the singular hyperplanes have been passed, and $\mathcal{T}_{\mu}^{\eta} \mathcal{F}_{\mu} f$ is then independent of $\eta$. We call it (that is, $\mathcal{T}_{\mu}^{\eta} \mathcal{F}_{\mu} f$ for $\eta$ sufficiently antidominant) a pseudo wave packet and denote it by $\mathcal{T}_{\mu} \mathcal{F}_{\mu} f$. It is a smooth $\mu$-spherical function on $(G / H)_{+}$, and it can be shown by taking the limit $\eta \rightarrow \infty$ that it vanishes outside a subset of $(G / H)+$ with compact closure in $G / H$.

We can now state the inversion formula for the Fourier transform $\mathcal{F}_{\mu}$.

Theorem 11, [16]. Let $f \in C_{c}^{\infty}(G / H ; \mu)$. Then

$$
\mathcal{T}_{\mu} \mathcal{F}_{\mu} f(x)=f(x)
$$

for all $x \in(G / H)_{+}$.

Theorem 11 is established by induction on $\operatorname{dim} \mathfrak{a}_{\mathrm{q}}$. The shift argument described earlier results in a formula expressing the difference $\mathcal{T}_{\mu} \mathcal{F}_{\mu} f-\mathcal{J}_{\mu} \mathcal{F}_{\mu} f$ of the pseudo wave packet and the wave packet as a sum of integrals of generalized residues. These residual integrals are by their construction only given as smooth functions on $(G / H)_{+}$; a crucial step is to extend them to smooth functions on $G / H$ (in fact, the residual integrals are not individually extended, only certain finite combinations extend). Let us indicate how the inversion formula and the smooth extension is obtained in the simplest case, when $\operatorname{dim} \mathfrak{a}_{\mathrm{q}}=1$ (in this case the result in fact follows already from the theory developed in [13]). The residual integrals, by which the pseudo wave packet $\mathcal{T}_{\mu} \mathcal{F}_{\mu} f$ differs from the wave packet $\mathcal{J}_{\mu} \mathcal{F}_{\mu} f$, are in this case just ordinary residues. Let $D$ be as in Theorem 10 , then the effect of $D$ is exactly to annihilate these residues, and hence $D \mathcal{T}_{\mu} \mathcal{F}_{\mu} f=D \mathcal{J}_{\mu} \mathcal{F}_{\mu} f=D f$ by Theorem 10 (ii). Thus the difference $\mathcal{T}_{\mu} \mathcal{F}_{\mu} f-f$, which is defined on $(G / H)_{+}$, is annihilated by $D$. Being also $K$-finite this difference is then an analytic function on $(G / H)_{+}$. However, since both $\mathcal{T}_{\mu} \mathcal{F}_{\mu} f$ and $f$ are compactly supported they agree on a non-empty open set, hence everywhere. In other words, the desired inversion formula holds. Moreover the sum of the residues, which we have now identified with $f-\mathcal{J}_{\mu} \mathcal{F}_{\mu} f$, extends smoothly to $G / H$.

The latter conclusion is the starting point for the inductive step that gives the proof for $\operatorname{dim} \mathfrak{a}_{\mathrm{q}}=2$. In this case there occur two kinds of residual integrals: those along one dimensional singular hyperplanes, and point residues, which are taken where the singular lines meet. Using some results from [15] and the smooth extension for $\operatorname{dim} \mathfrak{a}_{\mathrm{q}}=1$, the smooth extension is obtained for the sum of the residual line integrals. The argument for the inversion formula and the smooth 
extension of the sum of the point residues is now similar to the argument outlined above for $\operatorname{dim} \mathfrak{a}_{\mathrm{q}}=1$.

The inversion formula in Theorem 11 is the key to the Plancherel formula. More precisely, it is the version of it, in which the pseudo wave packet $\mathcal{T}_{\mu} \mathcal{F}_{\mu} f$ is replaced by the sum of the wave packet $\mathcal{J}_{\mu} \mathcal{F}_{\mu} f$ and the residual contributions. What remains for the Plancherel formula is essentially to identify these residual contributions in terms of the intermediate series and the most discrete series. The residues are taken along the singular hyperplanes of the functions involved, and at the intersections of these hyperplanes 'higher order' residues occur. The residues of the highest order are the point residues; it is the sum of these point residues that eventually becomes identified as the projection of $f$ to the discrete series. (In particular, if the discrete series is absent this means that the point residues cancel out.) First, however, the residues of lower order are identified in terms of generalized principal series representations induced from proper parabolic subgroups. It is here that we use Carmona's and Delorme's generalization [25] of Theorem 7. In particular, it follows that these lower dimensional residual integrals define Schwartz functions. Hence, as a consequence of the inversion formula, the sum of the point residues is also a Schwartz function. Since this is a finite sum of $\mathbb{D}(G / H)$-finite functions, one can conclude that it belongs to the discrete series.

\section{A Paley-Wiener theorem for $G / H$}

Let $\pi^{\prime}$ be the minimal polynomial satisfying the conclusion of Theorem 9 , and as before let $\pi$ be given by (29). We define the pre-Paley-Wiener space, $\mathcal{M}_{\mu}$ as the space of $\mathcal{V}(\mu)$-valued meromorphic functions $\varphi$ on $\mathfrak{a}_{\mathrm{q}}^{*}$, satisfying the following conditions:

(i) $\varphi(s \lambda)=C^{\circ}(s, \lambda) \varphi(\lambda)$, for all $s \in W_{\mathrm{q}}, \lambda \in \mathfrak{a}_{\mathrm{q}}^{*}$.

(ii) $\pi(\lambda) \varphi(\lambda)$ is holomorphic on a neighborhood of $\left(\mathfrak{a}_{\mathbf{q}}^{*} \mathbb{C}\right)_{-}$.

(iii) There exists a constant $R>0$ and for every $n \in \mathbb{N}$ a constant $C>0$ such that

$$
\|\pi(\lambda) \varphi(\lambda)\| \leq C(1+|\lambda|)^{-n} e^{R|\operatorname{Re} \lambda|}
$$

for all $\lambda \in\left(\mathfrak{a}_{\mathbf{q}}^{*} \mathbb{C}\right)_{-}$.

It can be seen that $\mathcal{F}_{\mu}$ maps $C_{c}^{\infty}(G / H ; \mu)$ into $\mathcal{M}_{\mu}$ (properties (i) and (ii) are straightforward consequences of (28) and Theorems 8 and 9 , whereas (iii) requires a more difficult estimate for $\left.E_{\mu}^{\circ}(\psi, \lambda)\right)$. It follows from the Paley-Wiener theorem of Helgason and Gangolli (see [46, Ch. IV, $\S 7$ ), that when $G / H$ is Riemannian and $\mu$ is the trivial $K$-type then $\mathcal{F}_{\mu}$ is a surjection onto the pre-Paley-Wiener space, as defined above for this special case. However in general one has to require further conditions on a function $\varphi \in \mathcal{M}_{\mu}$ before it belongs to $\mathcal{F}_{\mu}\left(C_{c}^{\infty}(G / H ; \mu)\right)$. Briefly put, the extra condition is that any existing relation between the normalized Eisenstein 
integrals and their derivatives (with respect to $\lambda$ ) should be reflected by a similar condition on $\varphi$. More precisely, we require that:

For all finite collections of $\partial_{1}, \ldots, \partial_{k} \in S\left(\mathfrak{a}_{\mathbf{q}}^{*}\right)$ (that is, constant coefficient differential operators on $\left.\mathfrak{a}_{\mathrm{q}}^{*}\right), \psi_{1}, \ldots, \psi_{k} \in \mathcal{V}(\mu)$ and $\lambda_{1}, \ldots, \lambda_{k} \in\left(\mathfrak{a}_{\mathbf{q}}^{*} \mathbb{C}\right)_{-}$, for which the relation

$$
\sum_{i=1}^{k} \partial_{i}\left[\pi(\lambda)\left\langle\psi \mid E_{\mu}^{\circ}\left(\psi_{i},-\bar{\lambda}\right)(x)\right\rangle\right]_{\lambda=\lambda_{i}}=0
$$

holds for every $\psi \in \mathcal{V}(\mu), x \in G / H$, we also have the relation

$$
\sum_{i=1}^{k} \partial_{i}\left[\pi(\lambda)\left\langle\varphi(\lambda) \mid \psi_{i}\right\rangle\right]_{\lambda=\lambda_{i}}=0
$$

The space of functions $\varphi \in \mathcal{M}_{\mu}$ satisfying this requirement is denoted $\mathrm{PW}_{\mu}$. It is clear from the definition $(26)$ of $\mathcal{F}_{\mu} f$ that it belongs to this space for $f \in$ $C_{c}^{\infty}(G / H ; \mu)$.

Theorem 12, [13], [16]. The $\mu$-spherical Fourier transform $\mathcal{F}_{\mu}$ is a bijection of $C_{c}^{\infty}(G / H ; \mu)$ onto the Paley-Wiener space $\mathrm{PW}_{\mu}$.

The injectivity of $\mathcal{F}_{\mu}$ is an immediate corollary of Theorem 10: If $\mathcal{F}_{\mu} f=0$ then $\mathcal{F}_{\mu} D f=\chi_{\mu}(D) \mathcal{F}_{\mu} f=0$, hence $D f=0$ by (ii), and hence $f=0$ by (i). The injectivity of $f \mapsto f^{\wedge}$ asserted earlier (below Theorem 6 ) is a consequence, by density of the $K$-finite functions in $C_{c}^{\infty}(G / H)$.

The proof of the surjectivity is based on the residue calculus that was described in the previous section. More precisely, given a function $\varphi \in \mathcal{M}_{\mu}$ one forms a pseudo wave packet from it as in (32), that is

$$
\mathcal{T}_{\mu} \varphi(x)=\left|W_{\mathrm{q}}\right| \int_{\eta+i \mathfrak{a}_{\mathrm{q}}^{*}} E_{+}(\lambda, x) \varphi(\lambda) d \lambda, \quad\left(x \in(G / H)_{+}\right)
$$

where $\eta$ is sufficiently antidominant. As before, one shows that $\mathcal{T}_{\mu} \varphi$ is supported on a subset of $(G / H)_{+}$with compact closure in $G / H$. The surjectivity of $\mathcal{F}_{\mu}$ is then a consequence of the following result.

Theorem 13, [16]. Assume that $\varphi \in \mathrm{PW}_{\mu}$. Then the pseudo wave packet $\mathcal{T}_{\mu} \varphi$ extends to a smooth function on $G / H$, belonging to $C_{c}^{\infty}(G / H ; \mu)$. Moreover,

$$
\mathcal{F}_{\mu} \mathcal{T}_{\mu} \varphi=\varphi
$$

The proof of this result is based on the same shift that was applied in the proof of Theorem 11. By this shift one expresses the pseudo wave packet $\mathcal{T}_{\mu} \varphi$ as the sum of the wave packet $\mathcal{J}_{\mu} \varphi$ and a residual part. Let us again outline the argument for the case when $\operatorname{dim} \mathfrak{a}_{\mathrm{q}}=1$ (in which case it is already given in [13]). By a clever 
idea introduced by Campoli, [22], for the split rank one group case and also used by Arthur, [1], there exists a function $f \in C_{c}^{\infty}(G / H ; \mu)$, the Fourier transform of which agrees with $\varphi$ (to some specified order of derivatives) at the (finitely many) locations where residues are taken. Hence the residual part of $\mathcal{T}_{\mu} \varphi$ is identical with the residual part of $\mathcal{T}_{\mu} \mathcal{F}_{\mu} f$, which was shown to extend smoothly in the proof of Theorem 11. Since also $\mathcal{J}_{\mu} \varphi$ is smooth on $G / H$ we conclude that $\mathcal{T}_{\mu} \varphi$ extends smoothly on $G / H$. We already mentioned that its support is compact, hence $\mathcal{T}_{\mu} \varphi \in$ $C_{c}^{\infty}(G / H ; \mu)$. In particular, it makes sense to form the Fourier transform in $(35)$. It follows from part of the proof of Theorem 10 that (35) holds when both sides are multiplied by the polynomial $\mu(D)$ (see [13, Lemma 21.10]), hence it also holds without this polynomial in front (as an identity between meromorphic functions).

For the Riemannian symmetric spaces the surjectivity of $\mathcal{F}_{\mu}$ (with an arbitrary $K$-type $\mu$ ) is a consequence of the Paley-Wiener theorem in [44], and for the group $G$ itself, considered as a symmetric space, it follows from [1], as mentioned earlier. Though it was inspired by [1], the proof outlined above differs from Arthur's treatise in several important respects. First of all, Arthur appeals to Harish-Chandra's Plancherel theorem, whereas here the idea is to prove both the Plancherel theorem and the Paley-Wiener theorem from the same kind of reasoning. In this respect the present proof is in the same spirit as that of Helgason and Rosenberg for $G / K$. Secondly, Arthur uses in the inductive argument a lifting theorem due to Casselman (see [1, Thm. II.4.1]). The use of this result (the proof of which seems still to be unpublished) is here replaced by the application of the theory of asymptotic families in $[15]$.

A partial Paley-Wiener theorem for $G / H$ was earlier obtained in [30]. The result, that a certain natural subspace of $\mathrm{PW}_{\mu}$ is contained in the range of the Fourier transform, was obtained by means of Theorem 3. This, as well as an application of the Paley-Wiener theorem to construct multipliers, is explained in [8].

Acknowledgement We are grateful to Patrick Delorme and Sigurdur Helgason for fruitful discussions, and to Toby Bailey and Tony Knapp for organizing a splendid conference.

\section{References}

1. J. Arthur, A Paley-Wiener theorem for real reductive groups, Acta Math. 150 (1983), 1-89.

2. E. P. van den Ban, Invariant differential operators on a semisimple symmetric space and finite multiplicities in a Plancherel formula, Ark. för Mat. 25 (1987), 175-187.

3. E. P. van den Ban, The principal series for a reductive symmetric space I. H-fixed distribution vectors, Ann. sci. Éc. Norm. Sup. 4, 21 (1988), 359-412.

4. E. P. van den Ban, The principal series for a reductive symmetric space II. Eisenstein integrals, J. Funct. Anal. 109 (1992), 331-441.

5. E. P. van den Ban, The action of intertwining operators on H-fixed generalized vectors in the minimal principal series of a reductive symmetric space, Indag. Math. (to appear). 
6. E. P. van den Ban, Induced representations and the Langlands classification, (this volume) (T. Bailey and A. W. Knapp, eds.).

7. E. P. van den Ban, J. Carmona, and P. Delorme, Paquets d'ondes dans l'espace de Schwartz d'un espace symétrique réductif, J. Funct. Anal. 139 (1996), 225-243.

8. E. P. van den Ban, M. Flensted-Jensen and H. Schlichtkrull, Basic harmonic analysis on pseudo-Riemannian symmetric spaces, Noncompact Lie Groups and some of their applications, (proceedings of the San Antonio NATO workshop, 1993) (E. A. Tanner and R. Wilson, eds.), vol. C 429, Kluwer, NATO ASI Series, 1994, pp. 69-101.

9. E. P. van den Ban and H. Schlichtkrull, Asymptotic expansions and boundary values of eigenfunctions on Riemannian symmetric spaces, J. reine und angew. Math. 380 (1987), 108-165.

10. E. P. van den Ban and H. Schlichtkrull, Multiplicities in the Plancherel decomposition for a semisimple symmetric space, Contemporary Math. 145 (1993), 163-180.

11. E. P. van den Ban and H. Schlichtkrull, Fourier transforms on a semisimple symmetric space, Invent. math. (to appear).

12. E. P. van den Ban and H. Schlichtkrull, Expansions for Eisenstein integrals on semisimple symmetric spaces, Arkiv för Mat. (to appear).

13. E. P. van den Ban and H. Schlichtkrull, The most continuous part of the Plancherel decomposition for a reductive symmetric space, Ann. of Math 145 (1997), 267-364.

14. E. P. van den Ban and H. Schlichtkrull, A residue calculus for root systems, Preprint, 1996.

15. E. P. van den Ban and H. Schlichtkrull, Analytic families of eigenfunctions on a reductive symmetric space, In preparation.

16. E. P. van den Ban and H. Schlichtkrull, The Paley-Wiener theorem and the Plancherel decomposition for a reductive symmetric space, In preparation.

17. M. Berger, Les espaces symétriques non compacts, Ann. Sci. École Norm. Sup. 74 (1957), $85-177$.

18. J.N. Bernstein, On the support of the Plancherel measure, J. Geom. Phys. 5 (1988), 663-710.

19. F. Bien, D-modules and spherical representations, Princeton U. P., Princeton, N. J., 1990.

20. N. Bopp and P. Harinck, Formule de Plancherel pour GL $(n, \mathbf{R}) / \mathrm{U}(p, q)$, J. reine und angew. Math. 428 (1992), 45-95.

21. J.-L. Brylinski and P. Delorme, Vecteurs distributions H-invariants pour les séries principales géneralisées d'espaces symétriques réductifs et prolongement méromorphe d'integrales d'Eisenstein, Invent. math. (1992), 619-664.

22. O. Campoli, The complex Fourier transform for rank-1 semisimple Lie groups, Thesis, Rutgers University (1976).

23. J. Carmona, Terme constant des fonctions tempérées sur un espace symétrique réductif, Preprint Luminy (1994).

24. J. Carmona and P. Delorme, Base méromorphe de vecteurs distributions H-invariants pour les séries principales géneralisées d'espaces symétriques réductifs. Equation fonctionelle, J. Funct. Anal. 122 (1994), 152-221.

25. J. Carmona and P. Delorme, Transformation Fourier pour les espaces symétriques réductifs, Preprint 1996.

26. E. Cartan, Sur la détermination d'un système orthogonal complet dans un espace de Riemann symétrique clos, Rend. Circ. Mat. Palermo 53 (1929), 217-252.

27. P. Delorme, Intégrales d'Eisenstein pour les espaces symétriques réductifs: Temperance, majorations. Petite matrice B, J. Funct. Anal. 136 (1996), 422-509.

28. P. Delorme, Troncature pour les espaces symétriques rd́uctifs, Preprint 1996.

29. P. Delorme, Formule de Plancherel pour les espaces symétriques réductifs, Preprint 1996.

30. P. Delorme and M. Flensted-Jensen, Towards a Paley-Wiener theorem for semisimple symmetric spaces, Acta Math. 167 (1991), 127-151.

31. J. Faraut, Distributions sphériques sur les espaces hyperboliques, J. Math. Pures Appl. 58 (1979), 369-444. 
32. M. Flensted-Jensen, Discrete series for semisimple symmetric spaces, Ann. of Math. 111 (1980), 253-311.

33. M. Flensted-Jensen, Analysis on Non-Riemannian Symmetric Spaces, Regional Conference Series in Math. 61, Amer. Math. Soc., Providence, 1986.

34. P. Harinck, Fonctions généralisées sphériques induites sur $G_{\mathbb{C}} / G_{\mathbb{R}}$ et applications, J. Funct. Anal. 103 (1992), 104-127.

35. P. Harinck, Inversion des intégrales orbitales et formule de Plancherel pour $G_{\mathbb{C}} / G_{\mathbb{R}}, \mathrm{C} . \mathrm{R}$. Acad. Sci. Paris 320 (1995), 1295-1298.

36. P. Harinck, Fonctions orbitales sur $G_{C} / G_{R}$. Formule d'inversion des intégrales orbitales et formule de Plancherel, Preprint 1996.

37. Harish-Chandra, Representations of semisimple Lie groups VI, Amer. J. Math. 78 (1956), 564-628.

38. Harish-Chandra, Discrete series for semisimple Lie groups, I, II, Acta Math. 113 (1965), 241-318; 116 (1966), 1-111.

39. Harish-Chandra, Harmonic analysis on real reductive groups, III. The Maass-Selberg relations, Ann. of Math. 104 (1976), 117-201.

40. G. Heckman and E. Opdam, Yang's system of particles and Hecke algebras, Ann. of Math. 145 (1997), 139-173.

41. G. Heckman and H. Schlichtkrull, Harmonic Analysis and Special Functions on Symmetric Spaces, Academic Press, 1994.

42. S. Helgason, A duality for symmetric spaces with applications to group representations, Adv. in Math. 5 (1970), 1-154.

43. S. Helgason, The surjectivity of invariant differential operators on symmetric spaces I, Ann. of Math. 98 (1973), 451-479.

44. S. Helgason, A duality for symmetric spaces with applications to group representations, II. Differential equations and eigenspace representations, Adv. in Math. 22 (1976), 187-219.

45. S. Helgason, Differential Geometry, Lie Groups, and Symmetric Spaces, Academic Press, New York, San Francisco, London, 1978.

46. S. Helgason, Groups and Geometric Analysis, Academic Press, Orlando, 1984.

47. S. Helgason, Geometric analysis on symmetric spaces, Amer. Math. Soc., 1994.

48. A. W. Knapp, Representation theory of semisimple groups. An overview based on examples, Princeton Univ. Press, 1986.

49. T. Matsuki, The orbits of affine symmetric spaces under the action of minimal parabolic subgroups, J. Math. Soc. Japan 31 (1979), 331-357.

50. T. Matsuki, A description of discrete series for semisimple symmetric spaces II, Adv. Studies in Pure Math 14 (1988), 531-540.

51. C. Moeglin and J.-L. Waldspurger, Spectral decomposition and Eisenstein series, Cambridge Univ. Press, 1995.

52. V. F. Molchanov, Plancherel's formula for pseudo-Riemannian symmetric spaces of rank 1 , Sov. Math., Dokl. 34 (1987), 323-326.

53. G. Ólafsson, Fourier and Poisson transformation associated to a semisimple symmetric space, Invent. math. 90 (1987), 605-629.

54. T. Oshima, A realization of semisimple symmetric spaces and construction of boundary value maps, Adv. Stud. Pure Math. 14 (1988), 603-650.

55. T. Oshima and T. Matsuki, A description of discrete series for semisimple symmetric spaces, Adv. Studies in Pure Math. 4 (1984), 331-390.

56. T. Oshima and J. Sekiguchi, Eigenspaces of invariant differential operators on an affine symmetric space, Invent. math. 57 (1980), 1-81.

57. R. Penney, Abstract Plancherel theorems and a Frobenius reciprocity theorem, J. Funct. Anal. 18 (1975), 177-190. 
58. J. Rosenberg, A quick proof of Harish-Chandra's Plancherel theorem for spherical functions on a semisimple Lie group, Proc. Amer. Math. Soc. 63 (1977), 143-149.

59. W. Rossmann, The structure of semisimple symmetric spaces, Canad. J. Math. 31 (1979), $157-180$.

60. H. Schlichtkrull, Hyperfunctions and harmonic analysis on symmetric spaces, Birkhäuser, Boston, 1984.

61. M. Sugiura, Fourier series of smooth functions on compact Lie groups, Osaka Math. J. 8 (1971), 33-47.

62. G. van Dijk and M. Poel, The Plancherel formula for the pseudo-Riemannian space GL $(n, \mathbf{R}) / \mathrm{GL}(n-1, \mathbf{R})$, Compos. Math. 58 (1986), 371-397.

63. D. Vogan, Irreducibility of discrete series representations for semisimple symmetric spaces, Adv. Studies in Pure Math. 14 (1988), 191-221.

64. N. Wallach, Real Reductive groups, I, II, Academic Press, 1988, 1992.

Department of Mathematics

University of Utrecht

P. O. Box 80010

NL 3508 TA Utrecht

The Netherlands

Department of Mathematics and Physics

The Royal Veterinary and Agricultural University

Thorvaldsensvej 40

DK 1871 Frederiksberg $C$

Denmark

Department of Mathematics

University of Copenhagen

Universitetsparken 5

DK 2100 Copenhagen

Denmark 